\title{
Untersuchung der Flusswasserinfiltration in voralpinen Schottern mittels Zeitreihenanalyse
}

\author{
Tobias Vogt $\cdot$ Eduard Hoehn $\cdot$ Philipp Schneider · Olaf A. Cirpka
}

Eingang des Beitrages: 24. 11.2008/Eingang des überarbeiteten Beitrages: 9.1.2009

(C) Springer-Verlag 2009

\begin{abstract}
Kurzfassung Grundwasserfassungen in der Nähe von Flüssen können durch die Infiltration von Flusswasser beeinflusst werden. Aus Sicht des Trinkwasserschutzes interessiert vor allem, welcher Anteil des geförderten Wassers aus dem Fluss stammt und wie lange das Flussinfiltrat im Grundwasserleiter verbleibt, bevor es gefördert wird. Hierzu können Markierversuche durchgeführt werden, die jedoch bei größeren Flüssen mit einem erheblichen Stoffeintrag verbunden sind. Als Alternative zu Markierversuchen stellen wir Methoden vor, um aus Zeitreihen der elektrischen Leitfähigkeit und der Temperatur quantitative Aussagen zu Mischungsverhältnissen und Aufenthaltszeiten abzuleiten. Wir empfehlen ein mehrstufiges Vorgehen bestehend aus: (1) einer qualitativen Analyse, (2) der spektralen Ermittlung des saisonalen Temperatur- und Leitfähigkeitsverlaufs, (3) einer Kreuzkorrelationsanalyse und (4) der nicht-parametrischen Dekonvolution der Zeitreihen. Wir wenden diese Methoden an drei Standorten im Grundwasserstrom des Thurtales im schweizerischen Mittelland an. An Standorten ohne gute Flussanbindung oder mit exfiltrierenden Verhältnissen können die aufwändigen Zeitreihenanalysen nicht angewendet werden, die Messreihen zeigen jedoch die entsprechenden Verhältnisse an. An Standorten mit dauerhafter Flussinfiltration kann aus den Zeitreihen die Durchbruchskurve eines
\end{abstract}

Dipl.-Geow. T. Vogt $(\varangle) \cdot$ Dr. E. Hoehn · Dr. P. Schneider

Eawag, das Wasserforschungsinstitut des ETH-Bereichs,

Abteilung Wasserressourcen und Trinkwasser,

Überlandstr. 133, 8600 Dübendorf, Schweiz

E-Mail: tobias.vogt@eawag.ch

Prof. Dr.-Ing. O.A. Cirpka

Eberhard-Karls-Universität Tübingen,

Zentrum für Angewandte Geowissenschaften (ZAG),

Sigwartstr. 10, 72076 Tübingen, Deutschland

E-Mail: olaf.cirpka@uni-tuebingen.de
Markierversuches rekonstruiert werden, ohne einen künstlichen Markierstoff in den Fluss geben zu müssen.

Investigation of bank filtration in gravel and sand aquifers using time-series analysis

Abstract Drinking-water wells in the vicinity of rivers may be influenced by infiltration of river water. In the context of drinking-water protection the decisive questions concern the fraction of river infiltrate in the pumped water and the residence time in the aquifer. For this purpose, tracer experiments may be performed. At larger rivers, however, such tests require the injection of large amounts of the tracer. As an alternative to artificial-tracer tests, we present methods in which time series of electric conductivity and temperature are used for quantitative statements regarding mixing ratios and residence times. We recommend a multistep approach consisting of: (1) a qualitative analysis of the time series, (2) a spectral filtering of the seasonal temperature and conductivity signals, (3) a cross-correlation analysis, and (4) a non-parametric deconvolution of the time series. We apply these methods to three sites in the aquifer of the Thur Valley in the Swiss Plateau. At sites without good connection between river and groundwater or where the river gains groundwater, the elaborate methods of timeseries analysis are not applicable, but the time series itself will reveal such conditions. At sites with continuous river water infiltration, we can reconstruct the breakthrough curve of a tracer test without releasing an artificial tracer into the river.

Keywords bank filtration t time-series analysis . temperature $\cdot$ electric conductivity $\cdot$ cross correlation . deconvolution 


\section{Einleitung}

$40 \%$ des Schweizer Trinkwassers wird durch aktive Förderung von zumeist alluvialem Grundwasser gewonnen (SVGW 2002). Viele bedeutende Trinkwasserfassungen befinden sich in Nachbarschaft zu Flüssen und fördern zu einem signifikanten Anteil Wasser aus Flussinfiltrat, das durch Aufenthaltszeiten von wenigen Tagen gekennzeichnet ist. Die Flüsse sind durch die Einleitung gereinigten Abwassers belastet, sodass die Nutzung des geförderten Grundwassers als Trinkwasser eine ausreichende Selbstreinigung des Infiltrats erfordert. In der Schweiz ist es deshalb üblich, die Anforderung an die engere Schutzzone S2 von zehn Tagen Aufenthaltszeit auch an die Infiltration von Flusswasser zu stellen (BUWAL 2004). Neben der Selbstreinigung gewährleistet die geforderte Aufenthaltszeit auch eine ausreichende Vorwarnzeit im Fall von Unfällen im Fluss.

Die Standardmethode zur Ermittlung von Fließzeitverteilungen zwischen einem Fluss und einem Pumpwerk besteht in der Durchführung eines Markierversuches, bei dem ein konservativer, leicht zu messender Markierstoff schlagartig in den Fluss gegeben und die Durchbruchskurve der Konzentration im Pumpwerk gemessen wird (z. B. Davis et al. 1980, Lin et al. 2003, Käss 2004). Der Markierversuch gibt jedoch nur die Verhältnisse während des Experiments wieder. In großen Flüssen ist es darüber hinaus erforderlich, eine große Masse des Markierstoffes einzusetzen.

Als Alternative zu Versuchen mit künstlichen Markierstoffen können natürliche Stoffe oder Eigenschaften als Tracer verwendet werden. Fließzeiten im Bereich mehrerer Jahre lassen sich mit der Tritium-Helium-Methode oder aus den Konzentrationen von anthropogenen Spurengasen wie Fluorchlorkohlenwasserstoffe und Schwefelhexafluorid abschätzen (z. B. Beyerle et al. 1999). Fließzeiten bis etwa zwei Wochen können aus Messungen der Radon-222Konzentration abgeschätzt werden (Hoehn \& von Gunten 1989, Hoehn 2007). Die Methode setzt jedoch voraus, dass die Emanation von Radon aus der Gesteinsmatrix räumlich gleichförmig ist, und dass mindestens eine der Proben die erreichbare Maximalkonzentration aufweist.

Natürliche Schwankungen physikalischer Wassereigenschaften lassen sich ebenfalls verwenden, um den Infiltrationsprozess von Flusswasser ins Grundwasser zu charakterisieren. Silliman \& Booth (1993) verwendeten hierzu Temperaturfluktuationen in einem Fluss und dem daran angeschlossenen Grundwasserleiter. Silliman et al. (1995) entwickelten eine analytische Lösung für den eindimensionalen konvektiven-konduktiven Wärmetransport im Grundwasser für beliebige Temperatursignale des infiltrierenden Wassers. Sheets et al. (2002) analysierten Piezometerhöhen, die Temperatur und die spezifische elektrische Leitfähigkeit in einem Testfeld in Cincinnati, Ohio, unter der Verwendung der Kreuzkorrelationsmethode. Constantz et al. (2003) vergli- chen Ergebnisse von künstlichen Tracertests mit der Analyse von Temperaturdaten beruhend auf der eindimensionalen Konvektions-Konduktions-Gleichung. Hatch et al. (2006) und Keery et al. (2007) analysierten Temperaturzeitreihen bei der Fließgewässerinfiltration auf der Grundlage einer Spektralanalyse der Konvektions-Konduktions-Gleichung.

Übersichtsartikel zur Verwendung der Temperatur als einfach zu messenden Tracer für den Fließgewässer-Grundwasser-Austausch wurden von Anderson (2005) und Constantz (2008) veröffentlicht. Der vorliegende Beitrag bezieht sich ausschließlich auf die Flusswasserinfiltration, sodass Methoden zur Quantifizierung der Grundwasserexfiltration mittels Temperaturmessungen nicht weiter behandelt werden (Conant 2004, Schmidt et al. 2006, 2007).

Für die Abschätzung der Infiltrationsgeschwindigkeit aus Temperaturdaten sind Zeitreihen erforderlich, die gut identifizierbare Schwankungen beinhalten. Hierbei überlagern sich drei Signale: Tagesgänge, Jahresgänge und witterungsbedingte Schwankungen mit einer typischen Dauer von mehreren Tagen bis wenigen Wochen. Die Dämpfung des Temperatursignals durch diffusionsartige Prozesse nimmt mit der Frequenz zu, sodass Tagesgänge häufig nur in sehr flussnahen Grundwasserbeobachtungsrohren zu beobachten sind, witterungsbedingte Signale in etwas weiterer Entfernung und saisonale Signale über weite Abstände. Die Schwierigkeit in der Auswertung saisonaler Temperaturschwankungen liegt darin, dass dasselbe Signal in allen Wässern oberflächennahen Ursprungs enthalten ist und deshalb eindeutiger Zusammenhang der gemessenen Zeitreihen mit der Fließgewässerinfiltration nicht gewährleistet ist (z. B. Hoehn \& Cirpka 2006). Eine weitere Einschränkung der Verwendung von Temperaturdaten ergibt sich daraus, dass die Wärmespeicherung in der Gesteinsmatrix zu berücksichtigen ist. Unter der Annahme des lokalen thermischen Gleichgewichts führt dies zu einer einfachen linearen Retardation. Falls jedoch große Körner in kleinen Zeiträumen (z.B. im Tagesrhythmus) erwärmt oder abgekühlt werden müssen, ist die Annahme des lokalen thermischen Gleichgewichts nicht mehr zulässig.

Cirpka et al. (2007) verwendeten Fluktuationen der spezifischen elektrischen Leitfähigkeit als natürlichen physikalischen Tracer für die Fließgewässerinfiltration. In dem betrachteten System, dem schweizerischen Mittellandsfluss Thur, treten Schwankungen der elektrischen Leitfähigkeit im Fluss in Folge von Niederschlagsereignissen im Einzugsgebiet auf. Dieses Signal wird in den Grundwasserkörper übertragen. Es kann grundsätzlich mit denselben Methoden ausgewertet werden wie das Temperatursignal. Im Methodenteil dieses Beitrages stellen wir die Kreuzkorrelationsmethode sowie die Auswertung mittels formfreier Dekonvolution vor.

In der Kreuzkorrelationsmethode wird der Korrelationskoeffizient von zwei Zeitreihen als Funktion der zeitlichen 
Verschiebung der beiden Datenreihen ermittelt, wobei ein Datensatz eventuell noch vor der Berechnung des Korrelationskoeffizienten geglättet wird (z. B. Sheets et al. 2002, Hoehn \& Cirpka 2006). Der Zeitversatz mit dem maximalen Korrelationskoeffizienten wird als charakteristische Zeit für die Signalübertragung von einem Messpunkt zum anderen interpretiert. Wie im Methodenteil erklärt wird, ist es sinnvoll für die optimale Kombination von Verschiebung und Glättung die zugehörige lineare Regression der Datenreihen durchzuführen.

Die Dekonvolution von zwei Zeitreihen geht davon aus, dass die beiden Zeitreihen als Eingabe- und Ausgabesignal eines linearen, zeitinvarianten Prozesses betrachtet werden können. Derartige Systeme sind durch eine Übertragungsfunktion, die dem Ausgangssignal bei pulsartiger Zugabe zum Zeitpunkt null entspricht, vollständig beschrieben (z.B. Jury 1982). Die Übertragungsfunktion entspricht damit einer normierten Durchbruchskurve für einen Markierversuch bei schlagartiger Zugabe. Die Anpassung von konstanten Transportkoeffizienten (effektive Geschwindigkeit und Dispersionskoeffizient) an die Zeitreihen kann als spezieller Fall einer Dekonvolution betrachtet werden, in dem die Form der Übertragungsfunktion vorgegeben der Lösung der Advektions-Dispersions-Gleichung für eine pulsartige Einleitung entspricht. Andere Parametrisierungen der Übertragungsfunktion sind denkbar, typischerweise in Form von Standardmodellen statistischer Verteilungen wie der Log-Normalverteilung, der Gamma-Verteilung usw. Die Schwierigkeit besteht häufig in der Wahl der besten Parametrisierung. In heterogenen Grundwasserleitern ist es durchaus möglich, dass ein Markierversuch zu Durchbruchskurven mit Eigenschaften führt, die den meisten parametrisierten Modellen widersprechen, z. B. MehrfachPeaks oder breite Peaks. Während solche Charakteristika beim Markierversuch offensichtlich werden, bleiben sie bei der Dekonvolution kontinuierlich fluktuierender Zeitreihen zunächst verborgen. Das heißt, in vielen Fällen kann eine zufriedenstellende Übereinstimmung zwischen Modell und Daten erreicht werden, obwohl die Parametrisierung eine nicht adäquate Form der Übertragungsfunktion vorschreibt.

In der formfreien Dekonvolution wird die Übertragungsfunktion als kontinuierliche Funktion ermittelt, ohne eine Parametrisierung vorzuschreiben. Im einfachsten Fall wird die Übertragungsfunktion diskretisiert und alle Einzelwerte durch Fehlerquadratminimierung ermittelt. Nach Übertragung der Ein- und Ausgangssignale in den Spektralraum entspricht die Dekonvolution einer einfachen Division, die jedoch leider nach Rückübertragung in reale Zeiten zu physikalisch unmöglichen Ergebnissen führen kann (Payn et al. 2008). Insbesondere führen Messfehler zu starken hochfrequenten Anteilen in der ermittelten Übertragungsfunktion und zu negativen Werten. Letztere sind grundsätzlich nicht möglich, weil weder Konzentration noch Wahrscheinlich- keitsdichten negative Werte annehmen können. Wenn man die Übertragungsfunktion als autokorrelierte nichtnegative Zeitfunktion betrachtet, ist es möglich ihre formfreie Ermittlung als ,geostatistisches“ inverses Problem zu definieren (Fienen \& Kitanidis 2006, Cirpka et al. 2007). Hierbei wird Nichtnegativität entweder durch die Wahl einer nichtnegativen A-priori-Verteilung in einem Bayesschen Ansatz (Fienen \& Kitanidis 2006) oder durch die Methode der Lagrangeschen Multiplikatoren erzwungen (Cirpka et al. 2007). Die Übertragungsfunktion wird hinreichend glatt durch die Forderung nach Autokorrelation.

Ziel dieses Artikels ist es, bestehende Zeitreihenmethoden zur Untersuchung von Flussinfiltrationsprozessen zu besprechen und ihre Einsatzmöglichkeit an drei Standorten im Thurtal zu diskutieren. Namentlich behandeln wir die Kreuzkorrelationsmethode und die Methode der formfreien Dekonvolution. Die Beispiele erfassen einen Standort, an dem diese Methoden gut eingesetzt und entsprechend miteinander verglichen werden konnten sowie zwei Standorte, bei denen Zeitreihen von Wasserstand, Temperatur und elektrischer Leitfähigkeit zwar für die Beurteilung des Fließgewässer-Grundwasser-Austausches eingesetzt werden konnten, der Einsatz der genannten Methoden jedoch nicht möglich war.

\section{Methoden}

\section{Datenerfassung}

Für die Erfassung von Zeitreihen des Wasserstandes, der Temperatur und der elektrischen Leitfähigkeit setzen wir Sensoren von KERN (Fieldlog FL-3-512) und STS (DL/ N 70) mit eingebauten Datenloggern ein. Für das Monitoring des Flusses wurden perforierte Rohre im Uferbereich installiert. Das Monitoring des Grundwassers erfolgt in teil- oder vollverfilterten PVC-Piezometern. Alle Datenlogger befinden sich in Kästen oberhalb der Hochwasserlinie. Für Grundwasseruntersuchungen ist eine Messfrequenz von einmal pro Stunde ausreichend. Im Fluss wurden die Daten viertelstündlich aufgenommen; die Zeitreihen wurden jedoch auf stündliche Messwerte reduziert, um die Frequenz aller Messungen zu vereinheitlichen. Fehlwerte in kurzen Ausfallzeiten, zum Beispiel während der Datenauslesung und Sensorenwartung, wurden durch lineare Interpolation ergänzt.

\section{Prinzipien der Auswertung}

Im Folgenden untersuchen wir die Zeitreihen von Wasserstand, Temperatur und spezifischer elektrischer Leitfähigkeit mit Methoden der Signalanalyse. Hierbei betrachten wir die Zeitreihe im Fluss als Eingangssignal $x(t)$ und diejenige 
in Grundwasserbeprobungsstellen als Ausgangssignal $y(t)$. Wir beschränken uns in der Analyse auf lineare, statistisch stationäre Systeme.

Da die Transportgleichungen für die Wärme und für einen gelösten konservativen Stoff linear sind, ist die Forderung der Linearität für Zeitreihen der Temperatur und der Konzentration eines konservativen Stoffes erfüllt. Die spezifische elektrische Leitfähigkeit ist jedoch ein Summenparameter, der sich aus den Konzentrationen verschiedener gelöster Salze ergibt, die nicht alle konservativ sind. In den von uns untersuchten Systemen nehmen die Mineralisation und damit die elektrische Leitfähigkeit des Grundwassers mit der Fließzeit zu. Wir analysieren deshalb nicht den Absolutwert der spezifischen elektrischen Leitfähigkeit, sondern die zeitliche Fluktuation um den Mittelwert. Näheres zur Trendentfernung wird weiter unten erklärt.

Eine Einschränkung der gewählten Auswerteverfahren ist durch die Forderung nach Stationarität gegeben. Sie besagt, dass das gleiche Eingangssignal unabhängig vom Zeitpunkt zum gleichen Ausgangssignal führt. Bei dynamischen Fluss-Grundwasser-Systemen ist diese Forderung streng genommen nicht erfüllt: Ein Markierversuch während eines Hochwassers würde wahrscheinlich zu einer anderen Durchbruchskurve im Grundwasser führen als ein Versuch bei Niedrigwasser. Die Verwendung von Methoden, die diese Effekte ausschließen, bedeutet, dass über verschiedene hydrologische Zustände gemittelt wird. Entsprechend sind Abweichungen zwischen Messungen und Modellvorhersage keine reinen Messfehler, sondern beinhalten auch Modellfehler, die sich aus der konzeptionellen Vereinfachung ergeben.

Auswertung mittels Kreuzkorrelation

Die Korrelation zweier Zeitreihen ist ein dimensionsloses $\mathrm{Ma} ß$ für den linearen Zusammenhang zwischen den Reihen. Sie quantifiziert, zu welchem Anteil die Varianz einer Zeitreihe auf die Varianz der anderen zurückgeführt werden kann. Für die Kreuzkorrelationsfunktion werden die beiden Zeitreihen um einen variablen Zeitversatz $\tau$ gegeneinander verschoben. Da der reine Zeitversatz noch nicht beschreiben kann, dass die Signale im Grundwasserleiter gegenüber dem Flusssignal geglättet sind, filtern wir das Eingangssignal $x(t)$ durch gleitende Mittelwertbildung über die Filterweite $w$ :

$$
\text { [1] } x_{f i l}(t, w)=\frac{1}{w} \int_{t-w / 2}^{t+w / 2} x(\xi) d \xi
$$

Die empirische Kreuzkorrelationsfunktion $r(\tau, w)$ zwischen dem gefilterten Eingangssignal $x_{f i l}(t, w)$ und dem Ausgangssignal $y(t)$ berechnet sich aus:

$$
r(\tau, w)=\frac{\int_{\tau+w / 2}^{T-w / 2}\left(x_{f l l}(t-\tau, w)-\mu_{x_{f l l}(w)}\right)\left(y(t)-\mu_{y}\right) d t}{(T-\tau-w) \sigma_{x_{f l l}(w)} \sigma_{y}}
$$

wobei davon ausgegangen wird, dass Zeitreihen von 0 bis $T$ bestehen, die jedoch durch die gleitende Mittelwertsbildung nur im Bereich $[w / 2, T-w / 2]$ ausgewertet werden können. $\mu_{x_{f i l}(w)}$ und $\mu_{y}$ sind die empirischen Mittelwerte sowie $\sigma_{x_{f i l}(w)}$ und $\sigma_{y}$ die empirischen Standardabweichungen der Zeitreihen $x_{f i l}(t, w)$ und $y(t)$ :

[3]

$$
\begin{aligned}
& \mu_{x_{f f l}(w)}=\frac{1}{T-w} \int_{w / 2}^{T-w / 2} x_{f i}(t, w) d t \\
& \mu_{y}=\frac{1}{T-w} \int_{w / 2}^{T-w / 2} y(t) d t \\
& \sigma_{x_{f f l}(w)}=\sqrt{\frac{1}{T-w} \int_{w / 2}^{T-w / 2}\left(x_{f i l}(t, w)-\mu_{x_{f l}(w)}\right)^{2} d t} \\
& \sigma_{y}=\sqrt{\frac{1}{T-w} \int_{w / 2}^{T-w / 2}\left(y(t)-\mu_{y}\right)^{2} d t}
\end{aligned}
$$

Die Kreuzkorrelationsfunktion $r(\tau, w)$ hängt sowohl vom Zeitversatz $\tau$ als auch der Filterweite $w$ ab. In unserer Analyse berechnen wir $r(\tau, w)$ für alle Kombinationen von $\tau$ und $w$ im Bereich zu erwartender Werte. Die optimale Kombination von $\tau$ und $w$ maximiert $r(\tau, w)$. Anschaulich kann der optimale Zeitversatz $\tau_{\text {opt }}$ im Fall von Leitfähigkeitszeitreihen als advektive Transportzeit interpretiert werden. Für den Fall der Temperatur handelt es sich um eine retardierte Transportzeit. Die optimale Filterweite $w_{\text {opt }}$ reflektiert Vermischungsprozesse in Fließrichtung.

Um aussagefähige Werte der Korrelationsfunktion zu erreichen, ist ein hinreichend großer Überlappungsbereich $T-\tau-w$ der beiden Zeitreihen erforderlich. Nur dann ist die zeitliche Mittelwertsbildung repräsentativ. Typischerweise wird die Korrelationsfunktion deshalb lediglich bis zu einem maximalen Zeitversatz $\tau_{\max }$ " $T-w$ ausgewertet.

Der Korrelationskoeffizient (gemäß Gl. 2) ist ein dimensionsloses Maß für die Güte eines linearen Modells zwischen den Signalen $x_{f i l}\left(t-\tau_{\text {opt }}, w_{\text {opt }}\right)$ und $y(t)$ :

$$
\text { [4] } y_{\text {reg }}(t)=a x_{f i l}\left(t-\tau_{\text {opt }}, w_{\text {opt }}\right)+b
$$

mit der Steigung $a$ und dem Achsenabschnitt $b$. Die Koeffizienten $a$ und $b$ können durch lineare Regression ermittelt werden, weshalb die Modellvorhersage für das Ausgangssignal auch mit $y_{\text {reg }}(t)$ bezeichnet ist. Wir empfehlen, die lineare Regression nach Ermittlung der optimalen Parameter $\tau_{\text {opt }}$ und $w_{\text {opt }}$ durchzuführen und die Regressionskoeffizienten auf ihre Plausibilität zu überprüfen.

Die Steigung $a$ kann als Wiederfindungsrate aufgefasst werden. Im Idealfall ergibt sich ein Wert von eins. Werte von $a<1$ deuten an, dass Fluktuationen im Eingangssignal nur zum Teil im Ausgangssignal wiedergefunden werden, was auf Verdünnungsprozesse hinweisen könnte. Für Werte von $a>1$ besteht keine physikalische Interpretation, weil die Ausgangssignale gegenüber den Eingangssignalen nicht verstärkt werden können. Typischerweise kann ein Wert von $a>1$ auf 
Artefakte zurückgeführt werden. Ein Beispiel hierfür wäre der Vergleich von zwei Zeitreihen, die weitgehend signalfrei sind, aber beide eine Spitze durch Störungen der Sensoren beinhalten. In einem solchen Fall kann eine beinah perfekte Kreuzkorrelation erreicht werden, die jedoch nichts mit den Prozessen während der Fließwasserinfiltration zu tun hat.

Der Achsenabschnitt $b$ beschreibt einen Versatz zwischen den beiden Signalen im Mittelwert. Der Idealwert beträgt null. Wie bereits geschildert, nimmt in unseren Systemen die Mineralisation des Grundwassers mit dem Fließabstand $\mathrm{zu}$, was zu Werten von $b>0$ im Fall von Leitfähigkeitszeitreihen führt. Im Fall von Wasserständen deutet $b \neq 0$ auf mittlere regionale hydraulische Gradienten hin.

Abbildung 1 zeigt die Analyse von Daten der elektrischen Leitfähigkeit in der Thur und im Pegel W2 am Standort Widen (Erläuterung des Standortes siehe unten) mittels Kreuzkorrelation und linearer Regression. Abbildung 1A zeigt die Originaldaten als Zeitreihen. Neben saisonalen Schwankungen sind kurzfristige Abnahmen der spezifi- schen elektrischen Leitfähigkeit über wenige Stunden bis Tage zu erkennen. Sie fallen mit hohen Wasserständen im Fluss zusammen und sind durch einen erhöhten Anteil niedrig mineralisierten meteorischen Wassers zu erklären. Offensichtlich wird dieses Signal auch ins Grundwasser übertragen. Abbildung 1B zeigt die Kreuzkorrelationsfunktion in Abhängigkeit vom Zeitversatz $\tau$ und der Filterweite $w$. Der Maximalwert des Korrelationskoeffizienten beträgt 0,93 bei $\tau_{\text {opt }}=24 \mathrm{~h}$ und $w_{\text {opt }}=49 \mathrm{~h}$. Die Abbildungen 1C und 1D zeigen Gegenüberstellungen der Zeitreihen im Fluss und im Grundwasser-Beobachtungsrohr. In Abbildung 1C sind die Originaldaten aufgetragen, wohingegen in Abbildung 1D das Eingangssignal um $\tau_{\text {opt }}$ verschoben und mit einem Rechteckfilter der Weite $w_{\text {opt }}$ geglättet wurde. Es ist ersichtlich, dass diese Modifikation des Eingangssignals zu einem stärker linearen Zusammenhang zwischen Ein- und Ausgangssignalen führt. Der Achsenabschnitt $b$ von $147 \mu \mathrm{S} /$ $\mathrm{cm}$ zeigt an, dass Reaktionen mit der Sedimentmatrix schon bei Aufenthaltszeiten von einem Tag zu einer signifikanten
Abb. 1 Beispielrechnung für die Kreuzkorrelation von Leitfähigkeitszeitreihen, hier Pegel W2 im Vorfeld des Pumpwerks Widen III in Felben-Wellhausen, TG, Schweiz. A: Zeitreihen der spezifischen elektrischen Leitfähigkeit; B: Kreuzkorrelationsfunktion in Abhängigkeit des Zeitversatzes $\tau$ und der Filterweite $w ; \mathrm{C}$ : gegenseitige Auftragung der Originaldaten der elektrischen Leitfähigkeit; $\mathrm{D}$ : gegenseitige Auftragung der Daten der elektrischen Leitfähigkeit nach Filterung und Zeitversatz mit optimalen Koeffizienten

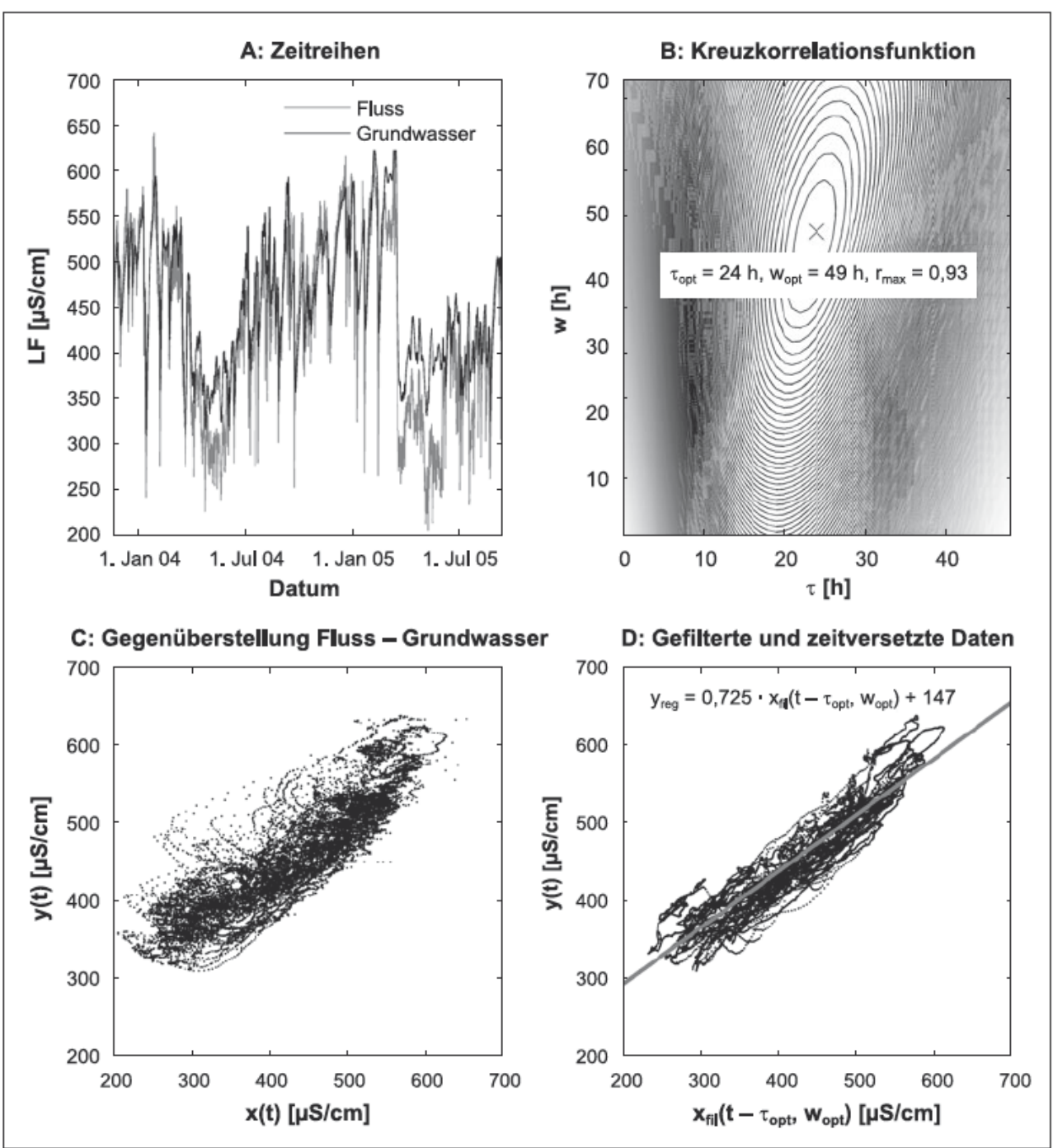


Mineralisation des infiltrierenden Wassers führen. Dies gilt insbesondere im späten Frühjahr. In der restlichen Jahreszeit besteht hingegen kein systematischer Unterschied in der elektrischen Leitfähigkeit von Fluss und flussnahem Grundwasser (siehe Abb. 1A). Die Unterschiede im Jahresgang erklären auch zum Teil den Wert der Steigung $a$ von 0,725 , der signifikant kleiner als eins ist.

\section{Saisonbereinigung}

Zeitreihen der Temperatur und der spezifischen elektrischen Leitfähigkeit beinhalten typischerweise Jahresgänge, Tagesgänge und Signale mit weniger stark ausgeprägten Wiederholungsrhythmen (zum Beispiel Änderungen der Wassertemperatur als Folge der Witterung). Für die Bestimmung von Fließzeiten im Bereich von Tagen bis wenigen Wochen ist das saisonale Signal meist nicht ausreichend. Bei Temperaturzeitreihen kommt erschwerend hinzu, dass das saisonale Signal nicht eindeutig auf die Fließgewässerinfiltration zurückgeführt werden kann. Dasselbe Signal wird dem Grundwasser auch aus regionaler Neubildung durch die ungesättigte Zone aufgeprägt, eventuell jedoch mit einem anderen Zeitversatz. Bezüglich der spezifischen elektrischen Leitfähigkeit zeigt das Beispiel in Abbildung 1, dass Unterschiede im Jahresgang eventuell eher auf Mineralisationsprozesse im Grundwasserleiter zurückzuführen sind als auf den rein physikalischen Stofftransport.

Vor diesem Hintergrund empfiehlt es sich, das saisonale Signal von den höherfrequenten Komponenten abzutrennen. Hierzu passen wir Sinus- und Cosinusfunktionen der Frequenzen 1/a, 2/a, 3/a und 4/a sowie einen konstanten Wert an alle Zeitreihen mittels multipler Regression an. Die harmonischen Komponenten bis 4/a ermöglichen es, einen nicht rein sinusartigen Jahresgang zu berücksichtigen. Das gefittete Modell der saisonalen Schwankung wird von der ursprünglichen Zeitreihe abgezogen. Die verbleibenden höherfrequenten Anteile beinhalten Tagesschwankungen, witterungsbedingte Schwankungen und Schwankungen infolge von Hochwasserereignissen.

\section{Auswertung mittels Dekonvolution}

Die Kreuzkorrelationsanalyse gibt zwar Hinweise auf den zeitlichen Versatz und die Glättung von Flusssignalen bei der Übertragung ins Grundwasser, die Daten werden jedoch im Sinne einer linearen Signalübertragung nicht ideal ausgewertet. Im Folgenden gehen wir davon aus, dass der Transport der betrachteten Größen (Temperatur, spezifische elektrische Leitfähigkeit, eventuell Wasserstand) durch Faltung beschrieben werden kann:

[5] $\quad y_{\text {conv }}(t)=\int_{0}^{\tau_{\max }} g(\tau) x(t-\tau) d \tau$
Hierbei ist $g(\tau)$ die Übertragungsfunktion des Systems, $\tau$ ist die Übertragungszeit, und $\tau_{\max }$ eine obere Schranke für $\tau$. Die Übertragungsfunktion oder Greensche Funktion beschreibt die Antwort des Systems auf einen Einheitspuls zum Zeitpunkt null. Dies entspricht der Einheitsganglinie in der Einzugsgebietshydrologie oder der normierten Durchbruchskurve eines konservativen Tracers bei schlagartiger Zugabe. Statistisch kann $g(\tau)$ als Wahrscheinlichkeitsdichtefunktion der Übertragungszeit interpretiert werden. Die Faltung eines bekannten Eingangssignals mit einer bekannten Übertragungsfunktion wird auch als Konvolution bezeichnet. Entsprechend wird die Ermittlung der Übertragungsfunktion aus bekannten Ein- und Ausgangssignalen als Dekonvolution angesprochen.

Die vorhergegangene Auswertung der Zeitreihen mittels Kreuzkorrelation kann ebenfalls im Kontext von Übertragungsfunktionen betrachtet werden. Der Zeitversatz, die gleitende Mittelwertsbildung und die lineare Regression entsprechen in Kombination einer parametrisierten Übertragungsfunktion mit Rechtecksform, die die Breite $w_{\text {opt }}$, das Flächenintegral $a$ und den Schwerpunkt $\tau_{o p t}$ aufweist:

[6] $g(\tau)= \begin{cases}\frac{a}{w_{o p t}} & \text { wenn } \tau_{\text {opt }}-\frac{w_{o p t}}{2} \leq \tau \leq \tau_{\text {opt }}+\frac{w_{o p t}}{2} \\ 0 & \text { sonst }\end{cases}$

Bei dieser Betrachtung wurde der Achsenabschnitt $b$ vernachlässigt. Das heißt, Unterschiede im Mittelwert lassen sich mithilfe der Übertragungsfunktion nicht erklären.

Es ist möglich, andere parametrisierte Modelle der Übertragungsfunktion an die Zeitreihen anzugleichen. Klassische Funktionen wären die Gammaverteilung (Luo et al. 2006), die lognormale Verteilung (Jury 1982) oder die fast identische inverse Gaußverteilung (Rao et al. 1981). Letztere entspricht der analytischen Lösung der eindimensionalen Advektions-Dispersionsgleichung mit konstanten Koeffizienten bei Zugabe und Detektion als Massenflusskonzentration (Kreft \& Zuber 1978). Parametrisierte Modelle sind durch wenige Koeffizienten vollständig charakterisiert, seien es physikalische Größen wie die Übertragungsgeschwindigkeit und der apparente Dispersionskoeffizient oder statistische Größen wie der Mittelwert, die Standardabweichung und die Schiefe. Grundsätzlich gilt, dass die Wahl des parametrisierten Modells die Form der Übertragungsfunktion festlegt. So beruhen die üblichen Modelle auf unimodalen Funktionen. Wenn die tatsächliche Übertragungsfunktion zwei Peaks aufweist, kann dies bei der Anpassung eines solchen parametrisierten Modells nicht abgebildet werden.

Eine Alternative zu parametrisierten Modellen besteht darin, formfreie Übertragungsfunktionen zu ermitteln (Fienen \& Kitanidis 2006, Cirpka et al. 2007). Hierbei werden die Übertragungszeit $\tau$ von null bis $\tau_{\max }$ mit derselben Schrittweite $\Delta t$ diskretisiert wie die Zeitreihen und für jeden diskreten Wert von $\tau$ ein Wert der Übertragungsfunkti- 
on bestimmt. Die Faltung in diskreter Form wird dann ein Matrix-Vektor-Produkt:

[7] $y_{\text {conv }}=X g$

mit der Matrix $X$, die mehrfach das Eingangssignal, jeweils pro Zeile um einen Eintrag versetzt, beinhaltet:

[8] $X_{i j}=\Delta t x_{i-j+1}$

und dem Vektor $g$ der diskreten Werte der Übertragungsfunktion. Ang stellen wir drei Anforderungen: (1) Das Produkt $X g$ gibt das gemessene Ausgangssignal $y$ im Sinne einer Fehlerquadratminimierung optimal wieder; (2) $g$ enthält keine negativen Einträge; und (3) $g(\tau)$ weist keine übermäßigen Fluktuationen auf. Letzteres wird gewährleistet, in dem wir $g(\tau)$ als eine zweiter-Ordnung intrinsische autokorrelierte Größe mit linearer Semivariogrammfunktion $\gamma_{g}$ betrachten:

$$
E\left[\frac{1}{2}(g(\tau+h)-g(\tau))^{2}\right]=\gamma_{g}(h)=\theta|h|
$$

Hierbei ist $\theta$ die Steigung der Semivariogrammfunktion und $\mathrm{E}[\cdot]$ ist der Erwartungswert. Für die Ermittlung der diskreten Übertragungsfunktionswerte $g$ wird diese als Summe des Mittelwertes $\beta$ und der Abweichungen $g$ beschrieben und die folgende Zielwertfunktion minimiert:

$$
\begin{gathered}
L\left(g^{\prime}, \beta \mid x, y\right)= \\
{[10] \frac{\left(y-X\left(g^{\prime}+u \beta\right)\right) \cdot\left(y-X\left(g^{\prime}+u \beta\right)\right)}{\sigma_{\text {fehl }}^{2}}-g^{{ }^{T}} \Gamma_{g g}^{-1} g^{\prime}}
\end{gathered}
$$

unter der Nebenbedingung $g=g^{\prime}+u \beta \geq 0$. Alle Elemente des Vektors $u$ bestehen aus Einsen. $\Gamma_{g g}$ ist die Semivariogrammmatrix der diskreten Übertragungsfunktionswerte, $\Gamma_{i j}$ $=\gamma_{g}\left(\tau_{i}-\tau_{j}\right) . \sigma_{\text {fenl }}$ ist der erlaubte Fehler. Die bedingte Minimierung erfolgt mit der Methode der Lagrange-Multiplikatoren (Lagrange 1788, S. 46-47). Die resultierenden Gleichungen werden von Cirpka et al. (2007) genau beschrieben. Eine mathematisch identische Formulierung des Optimierungsproblems bestünde darin, die Zielwertfunktion aus der Summe der gewichteten Fehlerquadrate (erster Term in Gleichung 10) und den gewichteten quadrierten Gradienten von $g(\tau)$ zu bilden (Tikhonov \& Arsenin 1977).

Wir ermitteln die Unsicherheit der ermittelten Übertragungsfunktion, indem wir konditionelle Realisationen generieren (Cirpka et al. 2007). Der optimale Wert für die Steigung $\theta$ der Semivariogrammfunktion wird mit der Expectation-Maximization-Methode bestimmt (McLachlan \& Krishnan 1997). Anschaulich kann diese Methode so erklärt werden, dass unkonditionelle und konditionelle Realisationen im gleichen Maß der Variogrammfunktion entsprechen sollen. Der Fehler $\sigma_{\text {fenl }}$ lässt sich aus den Daten abschätzen, in dem für die gewichteten Summe der quadrierten Residuen gefordert wird, dass sie ihrem Erwartungswert entsprechen (Press et al. 1992, Gl. 15.1.6).
Das Ergebnis der Dekonvolution ist die geschätzte Übertragungsfunktion vom Eingangs- zum Ausgangssignal mit seiner Unsicherheit. Dies entspricht der Verteilung der Fließzeit, die normalerweise durch Normierung der Durchbruchskurve eines schlagartig zugegebenen Markierstoffes ermittelt wird. Anstelle eines Markierversuches werden jedoch die Zeitreihen kontinuierlich schwankender GröBen ausgewertet. Die geschätzte Übertragungsfunktion $g(\tau)$ beinhaltet in Form ihres nullten zeitlichen Momentes $m_{0}(g)$ implizit eine Schätzung der Wiederfindungsrate:

$$
\text { [11] } m_{0}(g)=\int_{0}^{\tau_{\max }} g(\tau) d \tau
$$

Dies entspricht der Steigung $a$ in der linearen Regression des Ausgangssignals $y(t)$ gegenüber dem gefilterten und zeitlich versetzten Eingangssignal $x_{f i l}\left(t-\tau_{\text {opt }}, w_{\text {opt }}\right)$.

\section{Anwendungen im Thurtal}

\section{Hydrogeologische Situation}

Im untersuchten Gebiet fließt der voralpine Fluss Thur auf einer etwa $30 \mathrm{~km}$ langen und $2-3 \mathrm{~km}$ breiten Schotterebene von Osten nach Westen. Diese Schotterebene wird östlich durch einen Sporn aus Molassesandsteinen und -mergeln, und westlich durch eine späteiszeitliche Endmoräne begrenzt. Der Schotter wurde am Ende der letzten Eiszeit beim Rückzug des letzten Rheingletschers innerhalb weniger tausend Jahre geschüttet und ist für voralpine Verhältnisse ziemlich homogen. Er besteht aus einem Gemisch aus Kies und Sand mit wenig Feinanteil. Die Kornverteilung ähnelt dem (exponenziellen) Fuller-Gemisch mit Maximalkorn von etwa $30 \mathrm{~cm}$. Seine Mächtigkeit beträgt im östlichen Teil bis zu $25 \mathrm{~m}$. Er wird talabwärts zunehmend sandiger und keilt gegen das untere Ende der Ebene aus. Seine undurchlässige Basis besteht entweder aus Seelehm, Grundmoräne oder Molassegesteinen. Über dem Schotter liegt eine bis zu $5 \mathrm{~m}$ mächtige Schicht aus sandig-schluffigen Überschwemmungssedimenten. Der Schotter ist ein sehr guter Lockergesteins-Grundwasserleiter. Die Verteilung des aus Pumpversuchen in Grundwasserfassungen und Sondierbohrungen bestimmten Durchlässigkeitsbeiwertes hat eine negative Schiefe gegen die hohen Werte. Der häufigste Wert der gleichartig ausgewerteten Versuche beträgt $8 \cdot 10-3 \mathrm{~m} / \mathrm{s}$ (Mittelwert $\mu=5 \cdot 10-3 \mathrm{~m} / \mathrm{s}$, Varianz: $\sigma_{\log k_{f}}^{2}=0,4$; Amt für Umwelt Kanton Thurgau 2008).

Der Schotter wird von der Thur durchflossen. In ihrem Einzugsgebiet gibt es keinen See als Ausgleichsbecken, sodass die Thur ein besonders dynamisches Abflussregime aufweist (Niedrigwasserabfluss bis $3 \mathrm{~m}^{3} / \mathrm{s}$; mittlerer Abfluss: $20-50 \mathrm{~m}^{3} / \mathrm{s}$; Spitzen bis über $1.000 \mathrm{~m}^{3} / \mathrm{s}$; Bundesamt für Umwelt, Hydrographisches Jahrbuch: www.bafu.admin.ch/publikationen). Die Wasserqualität der Thur ist im Allgemeinen 


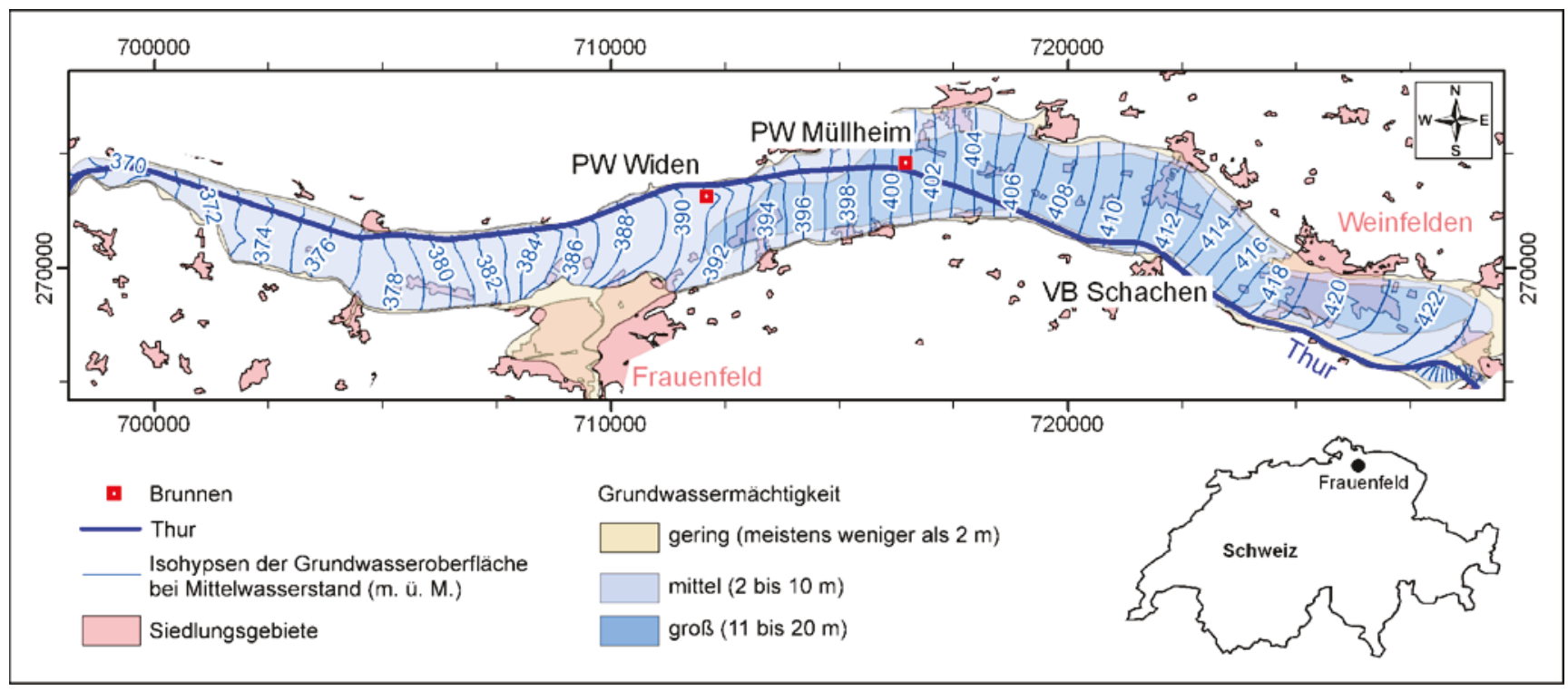

Abb. 2 Übersichtskarte über den Grundwasserstrom im Thurtal. Rechts- und Hochwerte in Schweizer Landeskoordinaten [m]. Reproduziert mit Bewilligung des Amts für Geoinformation des Kanton Thurgau vom 29.10.2008 und 30. 10.2008

Abb. 3 Zuströmbereich des Pumpwerks Widen III in Felben-Wellhausen, TG, Schweiz. Lage der Pegel und simulierte Grundwassergleichen. Rechtsund Hochwerte in Schweizer Landeskoordinaten $[\mathrm{m}]$

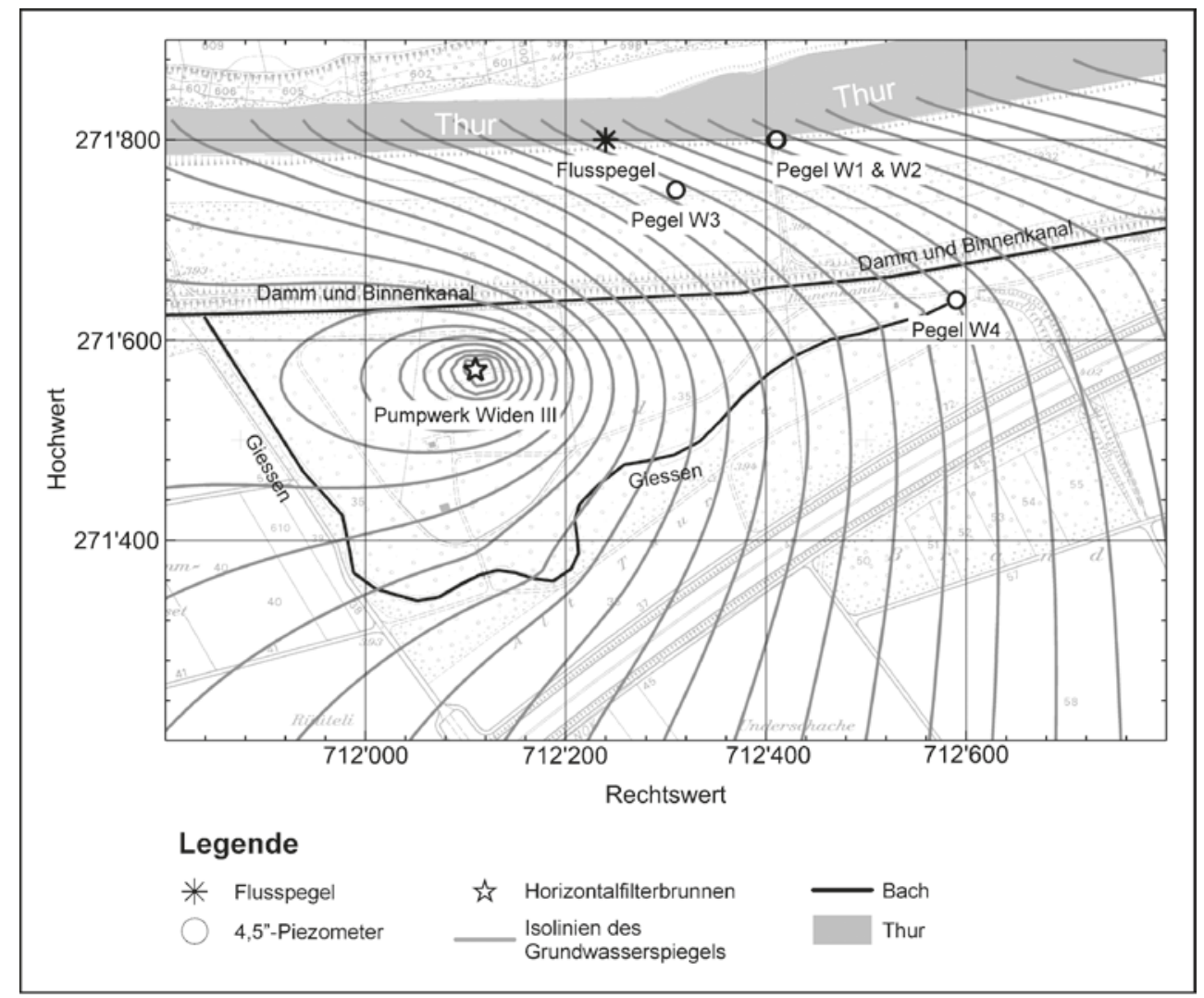

gut; sie wird aber durch landwirtschaftliche Einträge und Einleitungen von Abwasserreinigungsanlagen beeinträchtigt.

Im Thurtal fließt ein ergiebiger und stark für Trink- und Bewässerungswasser genutzter Grundwasserstrom (siehe
Abb. 2). Im oberen Bereich der Schotterebene liegt die Sohle der Thur über dem Grundwasserspiegel und reichert das Grundwasser über die ungesättigte Zone örtlich stark mit Infiltrat an. Stromabwärts füllt sich der Schotter zunehmend mit 
Grundwasser. Natürlicherweise würde das Grundwasser im unteren Bereich zurück in die Thur strömen. Beim Bau von Hochwasserschutz-Dämmen um 1890 wurden jedoch flussparallel Entwässerungskanäle („Binnenkanäle“) gegraben. Diese bewirken im unteren Talbereich auf weite Strecken eine Infiltration von Thurwasser, das sich als sehr junges Grundwasser in die Kanäle ergießt. Von neu gebildetem Grundwasser stammt etwa $85 \%$ aus der Thur, jedoch nur etwa $25 \%$ davon gelangt in den Bereich außerhalb der Binnenkanäle. Die Grundwassermächtigkeit beträgt im Allgemeinen 5-10m. Das Grundwasser fließt mit dem topographischen Gefälle und Geschwindigkeiten von $10-100 \mathrm{~m} / \mathrm{d}$ generell talparallel. Es ist im Allgemeinen etwas stärker mineralisiert und etwas härter als das Flusswasser (Kempf \& Labhart 2003).

\section{Pumpwerk Widen III, Felben-Wellhausen, TG}

Das Pumpwerk Widen III liegt im mittleren Bereich der Schotterebene, wo die Thur am nördlichen Talrand ver- läuft. In diesem Bereich ist der Kies 9-13 m mächtig, und die Grundwassermächtigkeit schwankt im Gebiet zwischen 5 und $10 \mathrm{~m}$. Der Flurabstand beträgt 1-3 m. Das Pumpwerk Widen III ist als Horizontalfilterbrunnen ausgebaut (Abb. 3, verändert aus Kempf \& Labhart 2003). Stränge mit geschlitzten Filtern von einer Gesamtlänge von $92 \mathrm{~m}$ liegen etwa 1 bzw. $3 \mathrm{~m}$ über dem Grundwasserstauer aus sehr schlecht durchlässigem Seelehm. Zwischen Thur und Pumpwerk verläuft ein Entwässerungskanal, der zumindest zeitweise sowohl etwas Uferfiltrat der Thur aufnimmt als auch etwas Wasser ins Grundwasser verliert. Der in Abbildung 3 dargestellte Zuströmbereich zum Brunnen weist für eine Förderleistung von 9.000 1/min eine Form auf, die auf eine Speisung sowohl von jungem und älterem Infiltrat als auch von landseitigem Grundwasser hindeutet.

Die Zeitreihen des Wasserstandes in der Thur, in den Grundwasserbeobachtungsstellen sowie im Pumpwerk sind hier nicht dargestellt. Sie weisen starke wetterbedingte Schwankungen auf. Die Pegel W1-W3 folgen praktisch un-

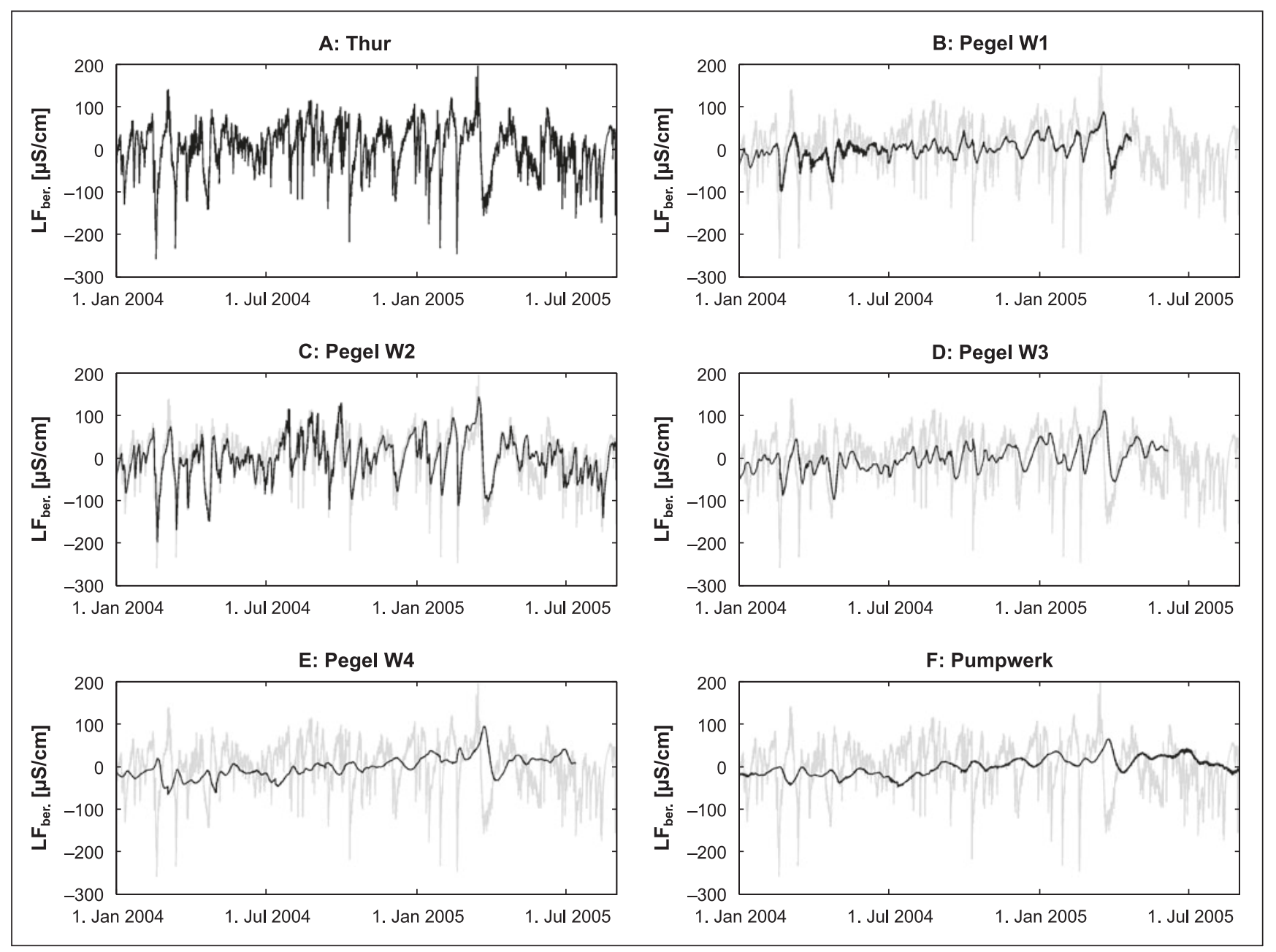

Abb. 4 Saisonbereinigte Zeitreihen der spezifischen elektrischen Leitfähigkeit im Zuströmbereich des Pumpwerks Widen III in Felben-Wellhausen, TG, Schweiz. Graue Linien: Zeitreihe der Thur zum Vergleich 
mittelbar den Wasserspiegelschwankungen in der Thur. Der Pegel W4 ist stark gedämpft. Der Wasserstand im Pumpwerk spiegelt hauptsächlich den Pumpenbetrieb wieder. In Zeiten ohne Förderung folgt dieser Wasserstand ebenfalls der Thur. Dies deutet bereits auf eine gute hydraulische Anbindung des Grundwasserleiters an die Thur hin.

Die ebenfalls nicht dargestellten Zeitreihen der Temperatur lassen sich wie folgt charakterisieren: Die Thur weist einen ausgeprägten Tagesgang der Temperatur auf, der in keinen der anderen Zeitreihen festgestellt werden kann. Die Pegel W1-W3 zeigen saisonale und witterungsbedingte Tempera- turschwankungen an, die auch im Thursignal enthalten sind. Die Temperaturzeitreihen des Pegels W4 sowie des Pumpwerks weisen nur noch das saisonale Signal auf. Dieser Befund steht damit im Einklang, dass die Dämpfung von Temperaturschwankungen mit der Frequenz zunimmt. Je weiter eine Messstelle vom Infiltrationspunkt entfernt ist, umso stärker wirkt die Grundwasserpassage als Tiefpassfilter.

Abbildung 4 zeigt die saisonbereinigten Zeitreihen der spezifischen elektrischen Leitfähigkeit in der Thur, in den Grundwasserbeobachtungsstellen sowie im Pumpwerk. Die in Abbildung 1 ersichtlichen Unterschiede im späten Früh-

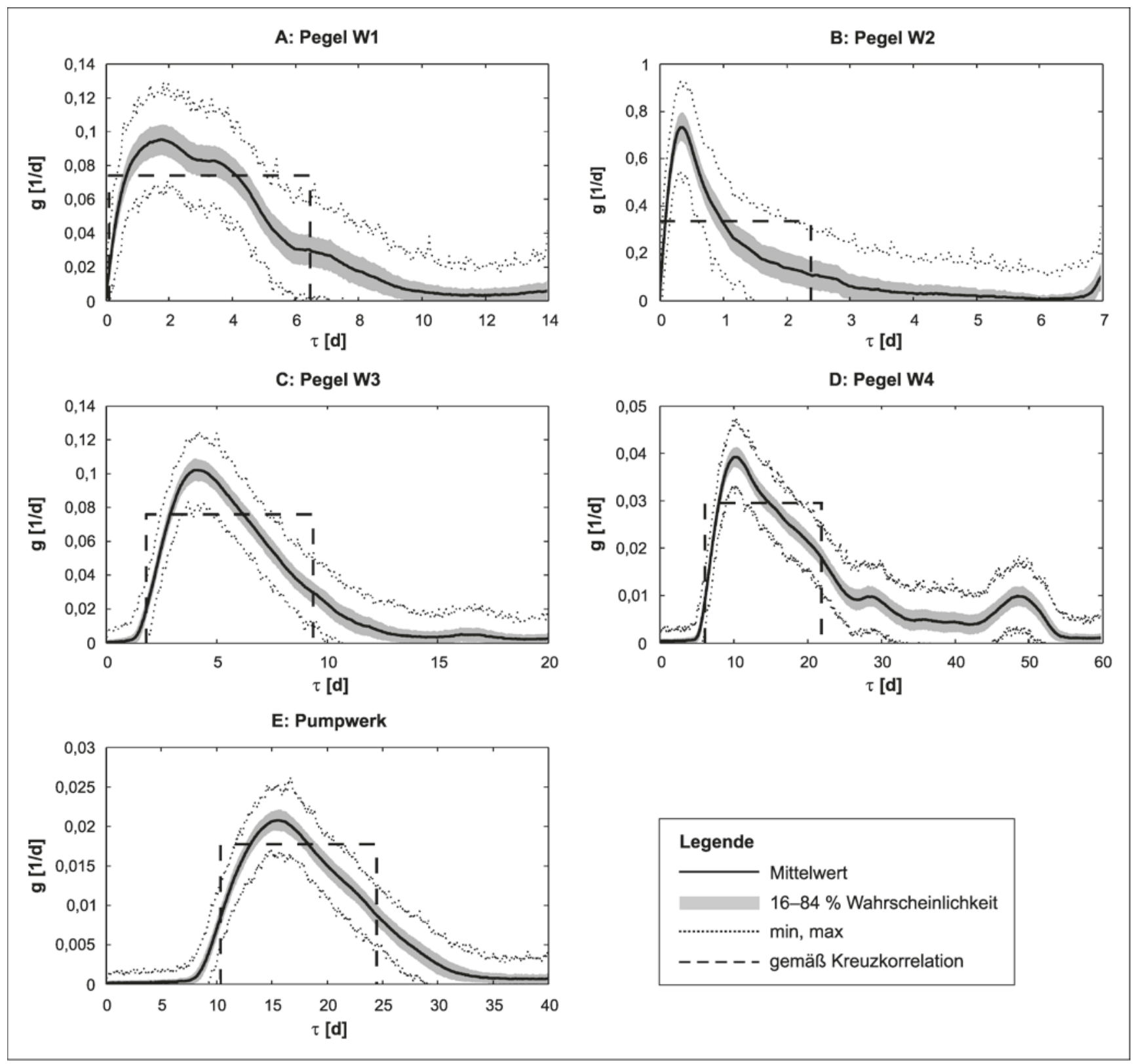

Abb. 5 Fließzeitverteilungen für die Pegel im Zuströmbereich des Pumpwerks Widen III in Felben-Wellhausen, TG, Schweiz. Grundlage: saisonbereinigte Daten. Das Wahrscheinlichkeitsintervall von 16-84\% entspricht \pm einer Standardabweichung für den Fall einer Log-Normalverteilung 
jahr wurden durch die Saisonbereinigung entfernt. Im Gegensatz zur nicht dargestellten Temperatur können Schwankungen der elektrischen Leitfähigkeit bis in das Pumpwerk beobachtet werden. Dies ist auf die niedrigere Diffusität der Stoffübertragung im Vergleich zur Wärmeübertragung zurückzuführen.

Abbildung 5 zeigt die aus den saisonbereinigten Zeitreihen der spezifischen elektrischen Leitfähigkeit abgeleiteten Übertragungsfunktionen oder Fließzeitverteilungen. Zum Vergleich beinhaltet Abbildung 5 auch die Übertragungsfunktionen, die sich aus der Kreuzkorrelationsanalyse ergeben. Tabelle 1 listet charakteristische Größen sowohl der Kreuzkorrelation als auch der Dekonvolution auf. Für die Pegel W1-W3 und das Pumpwerk kann eine gute Übereinstimmung zwischen Kreuzkorrelation und Dekonvolution bezüglich der Wiederfindungsrate ( $a$ bzw. $m_{0}(g)$ ) und der mittleren Fließzeit $\left(\tau_{\text {opt }}\right.$ bzw. $\left.t_{c}(g)\right)$ festgestellt werden. Die zeitliche Standardabweichung $\sigma_{g}$ der durch Dekonvolution ermittelten Übertragungsfunktion ist systematisch größer als die Standardabweichung $w / \sqrt{12}=0,29 w$ eines Rechtecksignals der Weite $w$. Dies ist im Wesentlichen darauf zurückzuführen, dass bei der Anpassung der Übertragungsfunktion an ein Rechtecksignal kein Tailing zugelassen wird. Für den Pegel W4 sind die Unterschiede zwischen den beiden Methoden am gravierendsten. Der Dekonvolutionsalgorithmus detektiert einen zweiten Peak in der Übertragungsfunktion, wohingegen das optimale Rechtecksignal ausschließlich mit dem ersten Peak zusammenfällt. Dies erklärt auch Unterschiede in statistischen Größen, die in Tabelle 1 aufgeführt sind.

Der thurseitige Zuströmbereich des Pumpwerks Widen III ist durch eine gute Anbindung des Grundwassers an den Fluss charakterisiert. Es herrschen praktisch immer infiltrierende Verhältnisse. Hydraulisch verhält sich der Grundwasserleiter dank der schlechter durchlässigen Schwemmsande im Hangenden wie ein gespannter Aquifer. Unter diesen Bedingungen stellen die Schwankungen der elektrischen Leitfähigkeit im Flusswasser einen guten natürlichen Tracer dar. Nach der vorliegenden Analyse besteht etwa ein
Viertel des geförderten Wassers aus frischem Thur-Infiltrat. Vergleichsabschätzungen auf der Grundlage von hydrochemischen Mischungsberechnungen ergaben einen Frischinfiltrat-Anteil von etwa einem Drittel (Hoehn et al. 2007). Ein signifikanter Durchbruch im Pumpwerk beginnt nach etwa 10 Tagen, was gerade dem Schweizer Richtwert für die innere Schutzzone S2 entspricht.

\section{Geplantes Pumpwerk Schachen, Weinfelden}

In einem Auenwald nahe eines Versuchsbrunnens (VB 1/99 in Abb. 6) ist der Bau eines Horizontalfilterbrunnens zur Versorgung der Stadt Weinfelden geplant. In diesem Bereich ist der Thurtal-Schotter 25-28 m mächtig. Das Grundwasser weist eine Mächtigkeit von 19-22 $\mathrm{m}$ auf und der Flurabstand beträgt 6-7 m. Der Grundwasserstauer besteht aus lehmigem Grundmoränen-Material.

Abbildung 7 zeigt die Zeitreihen des Wasserstandes, der Temperatur und der spezifischen elektrischen Leitfähigkeit der Pegel im Bereich Schachen von Ende Juli 2005 bis Oktober 2006 bzw. Januar 2007. Der Thur-Pegel P1 liegt in einer Restwasserstrecke des Flusses, weshalb die Dynamik des Wasserstandes bei Niedrigwasserabfluss unterbunden ist. Der Thur-Pegel P3 liegt unterhalb der Einleitung des Industriekanals Weinfelden und zeigt die volle Abflussdynamik. Aus Abbildung 7A ist ersichtlich, dass der Wasserstand in den Pegeln KB 3 und KB 4 sowie im Versuchsbrunnen VB 1/99 einer anderen Dynamik folgt als die Thur. Der Grundwasseranstieg im späten Frühjahr ist ein regionales Phänomen des Grundwasserleiters im gesamten östlichen Thurtal und kann auf die regionale Neubildung aus der ungesättigten Zone sowie auf verstärkte Fließgewässerinfiltration im Bereich Bürglen, TG, zurückgeführt werden. Der einzige Grundwasserpegel, der dem Wasserstand der Thur folgt, ist KB 5. Wie aus Abbildung 6 ersichtlich ist, liegt dieser Pegel auf dem rechten Vorland der Thur (Abstand zum Fluss $<10 \mathrm{~m}$ ).

Im Gegensatz zum Standort Widen enthalten die Zeitreihen der elektrischen Leitfähigkeit keine auswertbare Infor-

Tabel le 1 Auswertung der saisonbereinigten Zeitreihen der spezifischen elektrischen Leitfähigkeit im Bereich des Pumpwerks Widen III in Felben-Wellhausen, TG, Schweiz. $r_{\max }$ : maximaler Korrelationskoeffizient; $\tau_{\text {opt }}$ : optimaler Zeitversatz; $w_{\text {opt }}$ : optimale Filterweite; $a$ : Steigung der linearen Regression (Wiederfindungsrate gemäß Kreuzkorrelationsanalyse); $m_{0}(g)$ : nulltes Moment der Übertragungsfunktion (Wiederfindungsrate gemäß Dekonvolution); $t_{0}(g)$ : Fußpunkt der Übertragungsfunktion; $m_{c}(g)$ : Schwerpunkt der Übertragungsfunktion; $t_{m o d}(g)$ : Peak der Übertragungsfunktion; $\sigma_{g}$ : Standardabweichung der Übertragungsfunktion

\begin{tabular}{|c|c|c|c|c|c|c|c|c|c|}
\hline & \multicolumn{4}{|c|}{ Kreuzkorrelation } & \multicolumn{5}{|c|}{ Dekonvolution } \\
\hline & $r_{\max }$ & $\tau_{\text {opt }}$ & $w_{o p t}$ & $a$ & $m_{0}(\mathrm{~g})$ & $t_{0}(\mathrm{~g})$ & $t_{c}(\mathrm{~g})$ & $t_{\bmod }(\mathrm{g})$ & $\sigma_{g}$ \\
\hline Pegel W1 & 0,81 & $3 \mathrm{~d} 7 \mathrm{~h}$ & $6 \mathrm{~d} 9 \mathrm{~h}$ & 0,47 & 0,51 & instantan & $3 \mathrm{~d} 18 \mathrm{~h}$ & $1 \mathrm{~d} 18 \mathrm{~h}$ & $3 \mathrm{~d} 16 \mathrm{~h}$ \\
\hline Pegel W2 & 0,90 & $1 \mathrm{~d} 2 \mathrm{~h}$ & $2 \mathrm{~d} 15 \mathrm{~h}$ & 0,88 & 0,94 & instantan & $1 \mathrm{~d} 11 \mathrm{~h}$ & $8 \mathrm{~h}$ & $1 \mathrm{~d} 13 \mathrm{~h}$ \\
\hline Pegel W3 & 0,76 & $5 \mathrm{~d} 14 \mathrm{~h}$ & $7 \mathrm{~d} 13 \mathrm{~h}$ & 0,57 & 0,60 & $1 \mathrm{~d} 6 \mathrm{~h}$ & $6 \mathrm{~d} 2 \mathrm{~h}$ & $4 \mathrm{~d} 3 \mathrm{~h}$ & $3 \mathrm{~d} 22 \mathrm{~h}$ \\
\hline Pegel W4 & 0,55 & $14 \mathrm{~d} 2 \mathrm{~h}$ & $15 \mathrm{~d} 19 \mathrm{~h}$ & 0,47 & 0,68 & $4 \mathrm{~d} 22 \mathrm{~h}$ & $21 \mathrm{~d} 3 \mathrm{~h}$ & $10 \mathrm{~d} 7 \mathrm{~h}$ & $15 \mathrm{~d} 7 \mathrm{~h}$ \\
\hline Pumpwerk & 0,38 & $17 \mathrm{~d} 10 \mathrm{~h}$ & $14 \mathrm{~d} 3 \mathrm{~h}$ & 0,25 & 0,26 & $7 \mathrm{~d} 17 \mathrm{~h}$ & $18 \mathrm{~d} 2 \mathrm{~h}$ & $15 \mathrm{~d} 12 \mathrm{~h}$ & $10 \mathrm{~d} 0 \mathrm{~h}$ \\
\hline
\end{tabular}


Abb. 6 Pegel im Bereich des geplanten Pumpwerks Schachen in Weinfelden, TG, Schweiz. für Waldflächen. Rechts- und Hochwerte in Schweizer Landeskoordinaten $[\mathrm{m}]$. P1 und P3: Flusspegel; KB 3, KB 4; KB 5: Piezometer; VB 1/99: Schacht des Versuchsbrunnens Die Grauschattierungen stehen

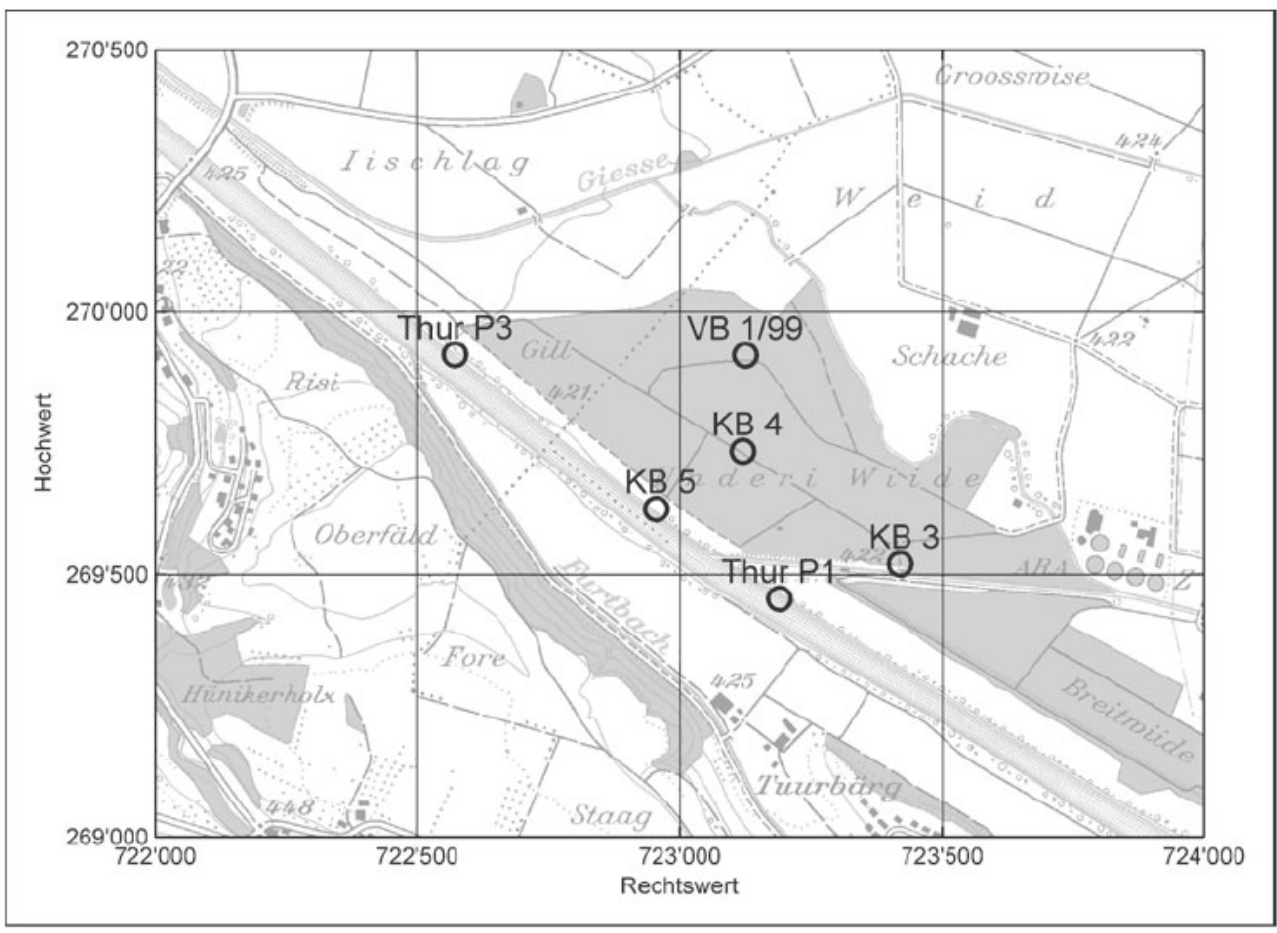

Tabelle 2 Spektrale Auswertung der saisonalen Temperaturschwankungen (Frequenz 1/a) für die Pegel im Bereich Schachen, Weinfelden, TG

${ }^{1}$ Filterstrecke $12-18 \mathrm{~m} \mathrm{u}$. GOK ${ }^{2}$ Filterstrecke $7-12 \mathrm{~m} \mathrm{u}$. GOK

\begin{tabular}{lccc}
\hline Pegel & Differenz Mittelwert $[\mathbf{K}]$ & Relative Amplitude [-] & Zeitversatz [d] \\
\hline KB 3 & 0,28 & 0,25 & 65 \\
KB 4a ${ }^{1}$ & 0,15 & 0,18 & 119 \\
${\text { KB } 4 b^{2}}^{2}$ & $-0,34$ & 0,23 & 107 \\
KB 5 & 0,30 & 0,15 & 119 \\
VB 1/99 & 0,85 & 0,15 & 145 \\
\hline
\end{tabular}

mation. Die Pegel KB 3 und KB 4 zeigen einen saisonalen Trend, der jedoch nur schwer auf saisonale Schwankungen der Leitfähigkeit in der Thur zurückgeführt werden kann. Das Leitfähigkeitssignal in dem der Thur nächsten Pegel KB 5 ist weitgehend flach bis auf eine Störperiode im März 2006. Die Leitfähigkeitsdaten aus dem Versuchsbrunnen VB 1/99 sind nicht dargestellt, weil vom Dezember 2005 bis zum März 2006 ein systematischer Fehler vorlag. In den ungestörten Perioden ähnelte der Verlauf den Daten der Pegel KB 3 und KB 4.

Die Zeitreihen der Temperatur in den Grundwasserbeobachtungsstellen weisen vornehmlich das saisonale Signal auf. Wie bei der elektrischen Leitfähigkeit, schwanken die Temperaturen im Pegel KB 5 während einer Störperiode im März 2006. Die Temperatur im Pegel KB 3, der relativ nah am Industriekanal liegt, fällt zu Beginn der Periode mit Frühjahrshochwässern um den 1. April 2006 zügig ab. Ein derartiges Verhalten ist bei keinem anderen Pegel zu beobachten.

Da weder die Zeitreihen der elektrischen Leitfähigkeit noch diejenigen der Temperatur höherfrequente Signalan- teile aufweisen, die für eine Analyse mittels Dekonvolution verwertet werden könnten, beschränkt sich unsere Auswertung auf das saisonale Temperatursignal. Hierzu wurde den Zeitreihen ein Sinus-Signal mit Jahresfrequenz und ein konstanter Mittelwert angepasst. Tabelle 2 beinhaltet die Differenz im Mittelwert, die relative Amplitude und den Zeitversatz des Jahressignals in den Grundwasserbeobachtungsstellen gegenüber desjenigen der Thur in Pegel P3. Hierbei zeigt sich, dass selbst Pegel KB 5, dessen Wasserstandsschwankungen denjenigen der Thur folgt, ein Temperaturmaximum aufweist, das gegenüber demjenigen der Thur um drei Monate versetzt ist. Unter der Annahme lokalen thermischen Gleichgewichts beträgt der Retardationskoeffizient für die konvektive Temperaturübertragung im Grundwasser 2,5-3,5 (Jäckli \& Ryf 1978). Das heißt, würde man annehmen, dass das Grundwasser vollständig aus Thur-Infiltrat besteht, betrüge die minimale Aufenthaltszeit des Wassers etwa 20 Tage (KB 3) und die maximale etwa zwei Monate (VB 1/99).

Wie aus Abbildung 7 ersichtlich ist, besteht fast immer ein Gefälle in der Piezometerhöhe von der Thur in den 


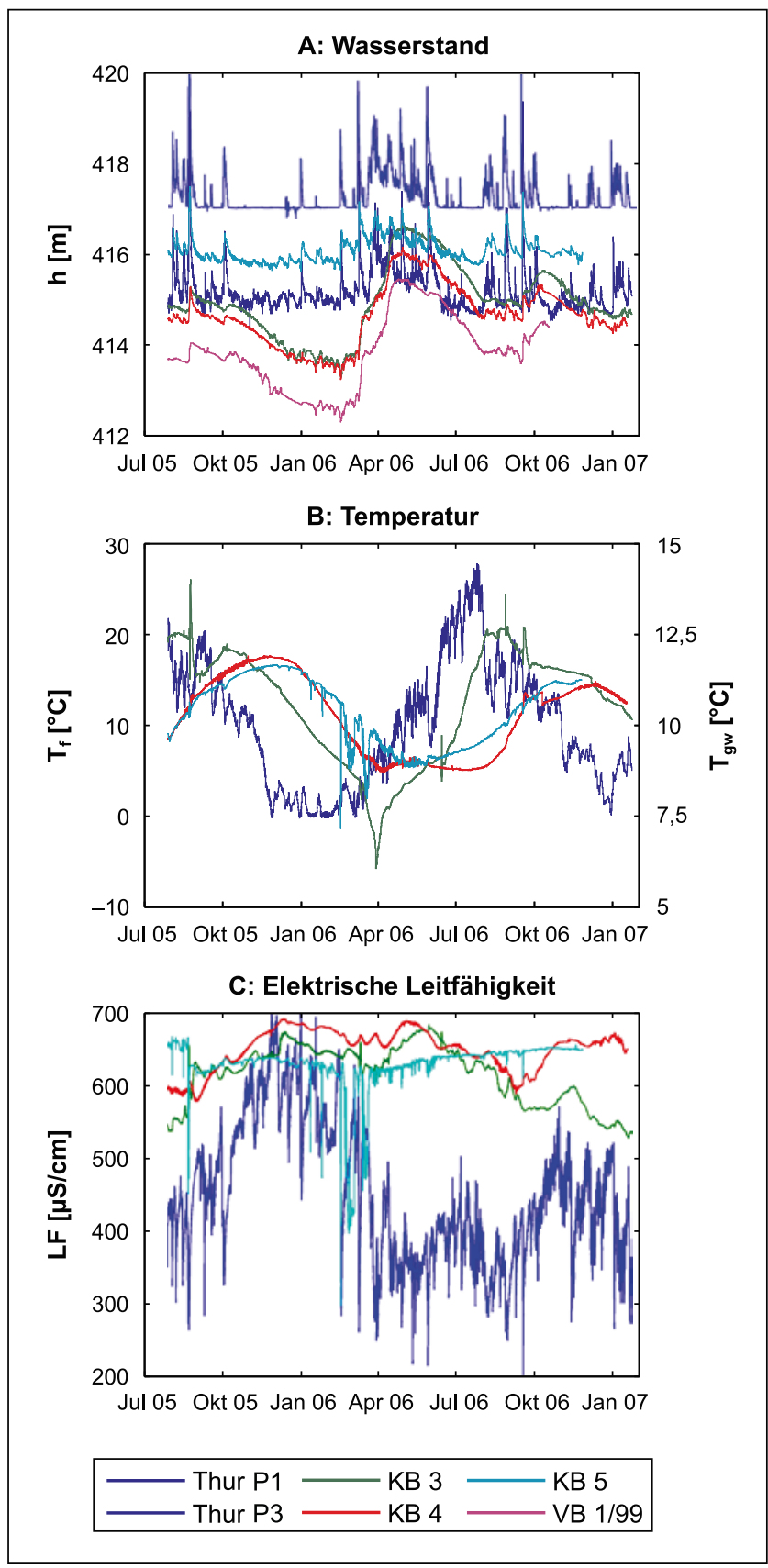

Abb. 7 Zeitreihen in Pegeln im Bereich des geplanten Pumpwerks Schachen in Weinfelden, TG, Schweiz. P1 und P3: Flusspegel; KB 3, KB 4; KB 5: Piezometer; VB 1/99: Schacht des Versuchsbrunnens. A: Wasserstand $[\mathrm{mNN}]$; B: Temperatur $\left[{ }^{\circ} \mathrm{C}\right]$; C: spezifische elektrische Leitfähigkeit $[\mu \mathrm{S} / \mathrm{cm}]$

Grundwasserkörper. Lediglich Pegel KB 5 reagiert unmittelbar auf Spiegelschwankungen der Thur. Alle anderen Pegel weisen den gleichen regionalen Trend auf, dessen Dynamik von der Grundwasserneubildung durch die ungesättigte Zone bestimmt wird. Trotz der starken Differenz in der Wasserspiegellage findet keine schnelle Infiltration statt. Selbst in Pegel KB 5 ergab sich eine starke zeitliche Ver- zögerung des Temperaturmaximums. Das heißt, es besteht eine schnelle Druckfortpflanzung in Thur-Nähe bei langsamer Strömungsgeschwindigkeit. Dies deutet auf eine niedrige Durchlässigkeit des anstehenden Gesteins hin. Das Bohrprofil von KB 5 dokumentiert tatsächlich schluffige Sande im wassererfüllten Bereich der Bohrung.

Aus den Zeitreihen lässt sich kein Einfluss der Thur auf das Grundwasser ableiten. Wir nehmen an, dass bei voller Förderungsleistung im Brunnen VB 1/99 eine Absenkung des Grundwasserspiegels zu erwarten wäre, der Infiltratanteil im geförderten Wasser jedoch klein wäre. Im Hinblick auf bakterielle Verunreinigungen der Thur oder des Industriekanals besteht insofern keine Gefahr für den Betrieb des Brunnens VB 1/99.

Pumpwerk „In der Thur“ Müllheim, TG

Das Pumpwerk „In der Thur“ (Abb. 8) befindet sich in Müllheim in einem Auenwald zwischen den beiden oben behandelten Standorten. Die Mächtigkeit des Schotterkörpers beträgt hier 17-18 m. Die Grundwassermächtigkeit beträgt $15-16 \mathrm{~m}$ mit einem Flurabstand von $2 \mathrm{~m}$. Der Grundwasserstauer wird aus sehr schlecht durchlässigem Seelehm gebildet. Das Pumpwerk „In der Thur“ ist als Vertikalfilterbrunnen ausgebaut. Die konzessionierte Entnahmemenge beträgt $2.6001 / \mathrm{min}$.

Abbildung 9 zeigt die Zeitreihen des Wasserstandes (A), der Temperatur (B) und der spezifischen elektrischen Leitfähigkeit (C) im Pumpwerk „In der Thur“" Müllheim (schwarz) und im Fluss (grau) von April 2007 bis Ende Oktober 2007. Weil alle Messdaten im Pumpwerk starke Schwankungen durch das mehrmals tägliche Ein- und Ausschalten der Pumpen aufweisen, sind in Abbildung 9 die Zeitreihen als gleitendes Mittel über 24 Stunden aufgetragen. In der Nähe des Pumpwerks „In der Thur“ selbst besteht kein Thur-Pegel. Deshalb handelt es sich bei den Thur-Daten um Zeitreihen vom Pegel im Bereich Widen (siehe Abb. 3). Bei allen Graphen ist zu beachten, dass die Pumpwerksdaten zur rechten Achse gehören und die Thur-Daten zur linken.

Der Wasserstand im Pumpwerk „In der Thur“ Müllheim korrespondiert gut mit dem Wasserstand in der Thur, wenngleich die Amplitude der Schwankungen auf etwa die Hälfte reduziert ist. Die Zeitreihen der Temperatur und der spezifischen elektrischen Leitfähigkeit zeigen hingegen kein kongruentes Verhalten. Der saisonale Anstieg der Temperatur im Pumpwerk beginnt erst etwa am 1. Juni und ist damit um drei Monate gegenüber dem Thur-Signal verspätet. Die mittlere spezifische elektrische Leitfähigkeit im Pumpwerk ist etwa doppelt so hoch wie diejenige in der Thur, was auf vergleichsweise lange Aufenthaltszeiten hinweist. Besonders auffällig sind Spitzen der elektrischen Leitfähigkeit am 9. 8., 31. 8., 5.9. und 19.9.2007, die jeweils mit hohen Wasserständen in der Thur zusammenfallen. Während dieser Er- 
Abb. 8 Lage des Pumpwerks „In der Thur" Müllheim, TG, Schweiz. Die Grauschattierungen stehen für Waldflächen. Rechts- und Hochwerte in Schweizer Landeskoordinaten [m]

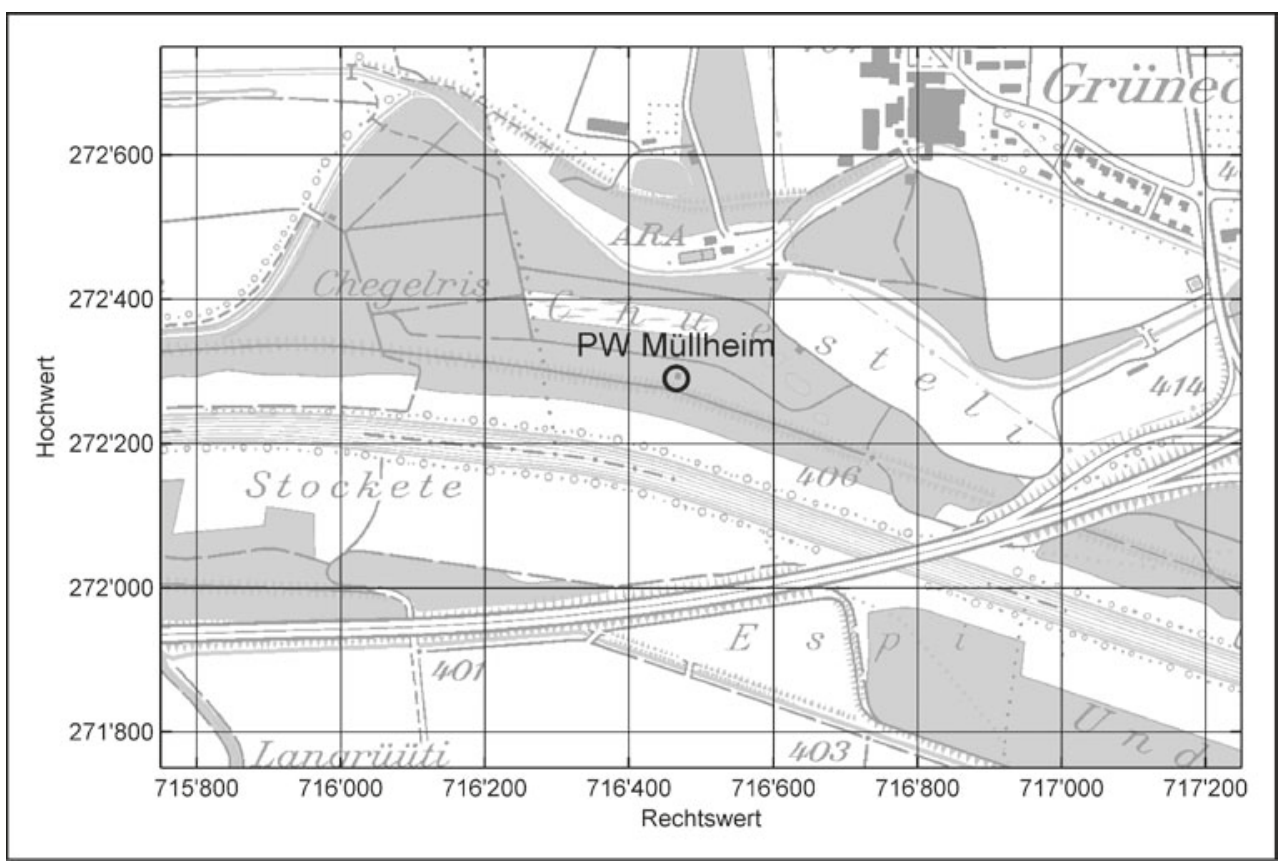

eignisse sinkt die elektrische Leitfähigkeit in der Thur, während sie im Pumpwerk ansteigt. Ein solches antikorreliertes Verhalten widerspricht vollkommen einer Situation, in der das Wasser aus der Thur auf schnellem Weg ins Pumpwerk gelangt.

Im Pumpwerk „In der Thur“ wurden am 6.12.2007 Wasserproben entnommen und auf gelöste Edelgase analysiert. Es konnte ein Tritium/Helium-Alter von zwei bis viereinhalb Jahren abgeschätzt werden. Dies bestätigt, dass das Pumpwerk „In der Thur“ kein frisches Thurinfiltrat fördert.

Ein regionales Modell für den Grundwasserstrom im Thurtal sagt für den Bereich des Pumpwerkes „In der Thur“ exfiltrierende Verhältnisse voraus, zumindest für die Seite rechts der Thur. Während des trockenen Sommers 2003 konnten in dem genannten Bereich Grundwasserzutritte im Uferbereich der Thur beobachtet werden. Dies ergibt ein konsistentes Bild. Das im Pumpwerk geförderte Wasser stammt entweder aus der regionalen Grundwasserneubildung oder aus der Infiltration von Thurwasser in einem Bereich weit oberstrom des Pumpwerkes. Das Wasser verweilt wenige Jahre im Grundwasserkörper bevor es im Pumpwerk gefördert wird.

Grundsätzlich können Wasserspiegelschwankungen sowohl in als auch entgegen der Strömungsrichtung fortgepflanzt werden. Deshalb steht die schnelle Druckausbreitung von der Thur ins Grundwasser nicht im Widerspruch zum Befund exfiltrierender Verhältnisse.

In Zeiten hohen Thurwasserstandes nimmt die Exfiltrationsrate ab, weil das hydraulische Gefälle zur Thur hin abnimmt. Dies verändert den dreidimensionalen Zuströmbereich des Pumpwerkes „In der Thur“. Wir vermuten, dass in einer solchen Situation tiefer gelegenes und damit älteres und stärker mineralisiertes Grundwasser vom Pumpwerk gefasst wird, das in Zeiten niedrigen Thurwasserstandes nicht ins Pumpwerk gelangt. Gleichzeitig sinkt die elektrische Leitfähigkeit in der Thur, weil der Anteil meteorischen Wassers zunimmt. Damit kann die Antikorrelation in den Zeitreihen der spezifischen elektrischen Leitfähigkeit als Rückstaueffekt interpretiert werden.

Selbst während des Hochwassers vom August 2007 fiel der Rückgang der Leitfähigkeit in der Thur mit einer $\mathrm{Zu}$ nahme im Pumpwerk zusammen. Wir folgern hieraus, dass selbst in dieser Hochwassersituation keine Umkehrung der Grundwasserströmungsrichtung erfolgte.

\section{Empfehlungen}

Zeitreihen kontinuierlich fluktuierender physikalischer Größen können als natürliche Tracer für die Charakterisierung der Flusswasserinfiltration eingesetzt werden. Je eindeutiger das Signal im Fluss ist, desto besser kann aus den parallel aufgenommenen Zeitreihen im Fluss und im Grundwasserbeobachtungspunkt die Übertragung des physikalischen Signals abgeleitet werden. Wir haben zwei Methoden zur Auswertung der Zeitreihen dargestellt: die rechnerisch und konzeptionell einfache Kreuzkorrelationsmethode und eine formfreie Dekonvolutionsmethode (Cirpka et al. 2007).

Das Beispiel des Standortes Widen zeigt, dass Schwankungen der spezifischen elektrischen Leitfähigkeit unter günstigen Umständen dazu benutzt werden können, nicht nur eine mittlere Verweilzeit zu bestimmen, sondern auch 


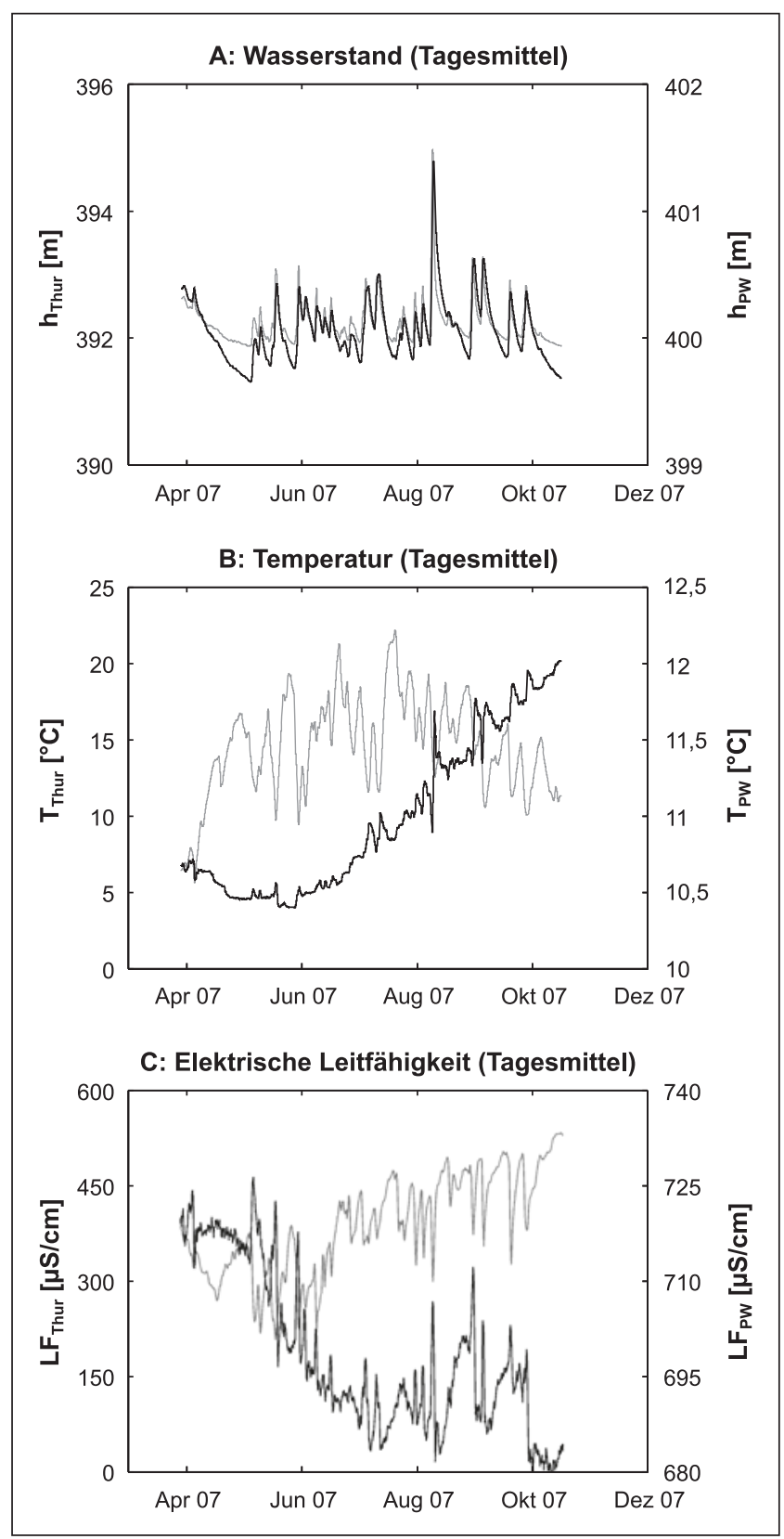

Abb. 9 Zeitreihen im Pumpwerk „In der Thur“ Müllheim (schwarz) und in der Thur beim Pumpwerk Widen (grau). A: Wasserstand $[\mathrm{mNN}]$; B: Temperatur $\left[{ }^{\circ} \mathrm{C}\right]$; C: spezifische elektrische Leitfähigkeit $[\mu \mathrm{S} / \mathrm{cm}]$

die Verteilung der Fließzeiten aus den erfassten Signalen abzuleiten. Dies ist nur mit der Dekonvolutionsmethode möglich. Einfachere Auswerteverfahren sollten jedoch der Anwendung der Dekonvolutionsmethode vorgelagert werden. Grundsätzlich empfehlen wir ein mehrstufiges Vorgehen. Im ersten Schritt werden die Daten gesichtet. Wenn die Zeitreihen kein nennenswertes Signal enthalten, erübrigt sich eine mathematisch komplizierte Auswertung (siehe Leitfähigkeitsdaten am Standort Schachen). Die erste
Durchsicht kann auch ergeben, dass eine Interpretation auf der Annahme der Flusswasserinfiltration physikalisch unmöglich ist (siehe anti-korrelierte Leitfähigkeitssignale am Standort Müllheim). Insofern können quantitativ nicht auswertbare Zeitreihen immer noch wertvolle Informationen über das qualitative Verhalten beinhalten. In einem zweiten Schritt empfehlen wir die Analyse saisonaler Trends. Am Standort Schachen zeigte das saisonale Temperatursignal an, dass ein schneller Infiltrationsprozess ausgeschlossen werden kann. In einem solchen Fall ist es nicht notwendig, weitere Methoden der Zeitreihenanalyse anzuwenden. Nach Entfernung des saisonalen Signals, schlagen wir in einem dritten Schritt die Durchführung der Kreuzkorrelationsanalyse vor. Das Verfahren ist leicht zu implementieren und gibt bereits gute Hinweise auf die mittlere Übertragungszeit sowie das Glättungsverhalten des Systems. Grundsätzlich empfehlen wir für die optimale Kombination von Zeitversatz und Filterweite die zur Korrelation gehörige lineare Regression durchzuführen und die ermittelten Koeffizienten auf ihre Plausibilität zu überprüfen. Nur wenn ein hoher Korrelationskoeffizient in der Kreuzkorrelationsanalyse erreicht werden kann, empfehlen wir in einem vierten Schritt die Durchführung der formfreien Dekonvolution, zunächst mit willkürlich festgesetztem Glätteparameter und ohne konditionelle Realisationen und dann, sofern die ermittelte Übertragungsfunktion plausibel erscheint, mit dem vollen Untersuchungsprogramm.

Danksagung Die dargestellte Arbeit wurde im Rahmen des Projektes „Assessment and Modeling of Coupled Hydrological and Ecological Dynamics in the Restored Corridor of a River (Ristored Corridor Dynamics): RECORD“ durchgeführt. Wir bedanken uns bei den Kollegen vom Amt für Umwelt des Kantons Thurgau, namentlich bei Marco Baumann, Romeo Favero und Robert Holzschuh, für die Bereitstellung der Daten.

\section{Literatur}

Amt für Umwelt Kanton Thurgau: 2. Thurgauer Thurkorrektion, Abschnitt Weinfelden Bürglen, Szenarienrechnungen. Bericht Simultec AG, unveröffentlicht (2008)

Anderson, M.P.: Heat as a ground water tracer.- Ground Water 43, 951-968 (2005)

Beyerle, U., Aeschbach-Hertig, W., Hofer, M., Imboden, D. M., Baur, H., Kipfer, R.: Infiltration of river water to a shallow aquifer investigated with ${ }^{3} \mathrm{H} /{ }^{3} \mathrm{He}$, noble gases and CFCs.- J. Hydrol. 220, 169-185 (1999)

BUWAL: Wegleitung Grundwasserschutz. Bundesamt für Umwelt, Wald und Landschaft, Bern (2004)

Cirpka, O.A., Fienen, M.N., Hofer, M., Hoehn, E., Tessarini, A., Kipfer, R., Kitanidis, P.K.: Analyzing bank filtration by deconvoluting time series of electric conductivity.- Ground Water $\mathbf{4 5}$, 318-328 (2007)

Conant, B.: Delineating and quantifying ground water discharge zones using streambed temperatures.- Ground Water 42, 243-257 (2004)

Constantz, J., Cox, M.H., Su, G. W.: Comparison of heat and bromide as ground water tracers near streams.- Ground Water 41, 647-656 (2003) 
Constantz, J.: Heat as a tracer to determine streambed water exchanges.Water Resour. Res., 44, W00D10, doi:10.1029/2008WR006996 (2008)

Davis, S. N., Thompson, G. M., Bentley, H.W., Stiles, G.: Ground-water tracers - a short review.- Ground Water 18, 14-23 (1980)

Departement für Bau und Umwelt Thurgau: Grundwassermodell Thurtal, Schlussbericht, unveröffentlicht (2008)

Fienen, M.N., Kitanidis, P. K.: A Bayesian geostatistical transfer function approach to tracer test analysis.- Water Resour. Res. 42(7), Art. No. W07426 (2006)

Hatch, C.E., Fisher, A.T., Revenaugh, J. S., Constantz, J., Ruehl, C.: Quantifying surface water-groundwater interactions using time series analysis of streambed thermal records: Method development.- Water Resour. Res. 42(10), Art. No. W10410 (2006)

Hoehn, E., von Gunten, H. R.: Radon in groundwater: a tool to assess infiltration from surface waters to aquifers.- Water Resour. Res. 25, 1795-1803 (1989)

Hoehn, E., Cirpka, O.A.: Assessing hyporheic zone dynamics in two alluvial flood plains of the Southern alps using water temperature and tracers.- Hydrol. Earth Sys. Sci. 10, 553-563 (2006)

Hoehn, E.: Überwachung der Auswirkung von Flussaufweitungen auf das Grundwasser mittels Radon.- Grundwasser 12, 66-72 (2007)

Hoehn, E., Cirpka, O.A., Hofer, M., Zobrist, J., Kipfer, R., Baumann, M., Scholtis, A., Favero, R.: Untersuchungsmethoden der Flussinfiltration in der Nähe von Grundwasserfassungen.- gwa Gas Wasser Abwasser 2007, 497-505 (2007)

Jäckli, H., Ryf, W.: Die Grundwasserverhältnisse im unteren aargauischen Aaretal.- Wasser, Energie, Luft, 70 (3/4), 53-61 (1978)

Jury, W.A.: Simulation of solute transport using a transfer function model.- Water Resour. Res. 18, 363-368 (1982)

Käss, W.: Geohydrologische Markierungstechnik. 2. Auflage.- 557 S.; Bornträger, Berlin (2004)

Keery, J., Binley, A., Crook, N., Smith, J.W.N.: Temporal and spatial variability of groundwater-surface water fluxes: Development and application of an analytical method using temperature time series.- J. Hydrol. 336, 1-16 (2007)

Kempf, Th., Labhart, W.: Die Grundwasserverhältnisse im Kanton Thurgau, Erläuterungen zur Grundwasserkarte 1:25.000. Dr. H. Jäckli AG, Amt für Umwelt Thurgau.- 88 S. (2003)

Kreft, A., Zuber, A.: On the physical meaning of the dispersion equation and its solutions for different initial and boundary conditions.Chem. Eng. Sci. 33, 1471-1480 (1978)

Lagrange, J. L.: Mécanique Analytique; Desaint, Paris (1788)
Lin, A.Y.C., Debroux, J.F., Cunningham, J.A., Reinhard, M.: Comparison of rhodamine WT and bromide in the determination of hydraulic characteristics of constructed wetlands.- Ecol. Eng. 20, 75-88 (2003)

Luo, J., Cirpka, O.A., Fienen, M.N., Wu, W., Mehlhorn, T.L., Carley, J., Jardine, P. M., Criddle, C. S., Kitanidis, P. K.: A parametric transfer function methodology for analyzing reactive transport in nonuniform flow.- J. Contam. Hydrol. 83, 27-41 (2006)

McLachlan, G.J., Krishnan, T.: The EM algorithm and extensions; John Wiley, New York (1997)

Payn, R.A., Gooseff, M.N., Benson, D.A., Cirpka, O.A., Zarnetske, J.P., Bowden, W.B., McNamara, J.P., Bradford, J.H.: Comparison of instantaneous and constant-rate stream tracer experiments through non-parametric analysis of residence time distributions.Water Resources Research 44(6), Art. No. W06404 (2008)

Press, W.H., Teukolsky, S.A., Vetterling, W.T., Flannery, B. P.: Numerical Recipes in FORTRAN 77: The art of scientific computation. 2nd edition.- Cambridge University Press, New York (1992)

Rao, P.V., Portier, K.M., Rao, P.S.C.: A stochastic approach for describing convective-dispersive solute transport in saturated porous-media.- Water Resour. Res., 17 (4), 963-968 (1981)

Schmidt, C., Bayer-Raich, M., Schirmer, M.: Characterization of spatial heterogeneity of groundwater-stream water interactions using multiple depth streambed temperature measurements at the reach scale.- Hydrology and Earth System Sciences 10, 849-859 (2006)

Schmidt, C., Conant, B., Bayer-Raich, M., Schirmer, M.: Evaluation and field-scale application of an analytical method to quantify groundwater discharge using mapped streambed temperatures.Journal of Hydrology 347, 292-307 (2007)

Sheets, R.A., Darnera, R.A., Whitteberry, B.L.: Lag times of bank filtration at a well field, Cincinnati, Ohio, USA.- J. Hydrol. 266, 162-174 (2002)

Silliman, S.E., Booth, D. F.: Analysis of time-series measurements of sediment temperature for identification of gaining vs losing portions of Juday-Creek, Indiana.- J. Hydrol. 146, 131-148 (1993)

Silliman, S.E., Ramirez, J., McCabe, R.L.: Quantifying downflow through creek sediments using temperature time-series - one-dimensional solution incorporating measured surface-temperature.J. Hydrol. 167, 99-119 (1995)

SVGW: Infoblatt TWI 12. Schweizerischer Verein des Gas- und Wasserfachs; Zürich (2002)

Tikhonov, A. N., Arsenin, V.A.: Solution of Ill-posed problems; Winston \& Sons, Washington (1977) 